\title{
Extragalactic Astrophysics With Next-Generation CMB Experiments
}

\author{
Gianfranco De Zotti ${ }^{1 *}$, Matteo Bonato ${ }^{1,2,3}$, Mattia Negrello ${ }^{4}$, Tiziana Trombetti ${ }^{2}$, \\ Carlo Burigana ${ }^{2,5,6}$, Diego Herranz ${ }^{7,8}$, Marcos López-Caniego ${ }^{9}$, Zhen-Yi Cai ${ }^{10}$, \\ Laura Bonavera ${ }^{11}$ and Joaquin González-Nuevo ${ }^{11}$
}

\begin{abstract}
${ }^{1}$ Osservatorio Astronomico di Padova, Istituto Nazionale di Astrofisica, Padova, Italy, ${ }^{2}$ Istituto di Radioastronomia, Istituto Nazionale di Astrofisica, Bologna, Italy, ${ }^{3}$ Italian ALMA Regional Centre, Istituto Nazionale di Astrofisica, Bologna, Italy, ${ }^{4}$ School of Physics and Astronomy, Cardiff University, Cardiff, United Kingdom, ${ }^{5}$ Dipartimento di Fisica e Scienze della Terra, Università di Ferrara, Ferrara, Italy, ${ }^{6}$ Istituto Nazionale di Fisica Nucleare, Sezione di Bologna, Bologna, Italy, ${ }^{7}$ Instituto de Física de Cantabria, Spanish National Research Council-University of Cantabria, Santander, Spain, ${ }^{8}$ Departamento de Física Moderna, Universidad de Cantabria, Santander, Spain, ${ }^{9}$ ESAC, Madrid, Spain, ${ }^{10}$ CAS Key Laboratory for Research in Galaxies and Cosmology, Department of Astronomy, University of Science and Technology of China, Hefei, China,

${ }^{11}$ Departamento de Física, Universidad de Oviedo, Oviedo, Spain
\end{abstract}

\section{OPEN ACCESS}

Edited by:

Maurizio Tomasi,

University of Milan, Italy

Reviewed by:

Joaquin Vieira

University of Illinois at

Urbana-Champaign, United States

Rahul Datta,

Johns Hopkins University,

United States

*Correspondence:

Gianfranco De Zott

gianfranco.dezotti@inaf.it

Specialty section:

This article was submitted to

Cosmology

a section of the journal Frontiers in Astronomy and Space

Sciences

Received: 29 March 2019

Accepted: 11 July 2019

Published: 06 August 2019

Citation:

De Zotti G, Bonato M, Negrello M, Trombetti T, Burigana $C$, Herranz $D$, López-Caniego M, Cai Z-Y,

Bonavera $L$ and González-Nuevo J (2019) Extragalactic Astrophysics With Next-Generation CMB Experiments.

Front. Astron. Space Sci. 6:53.

doi: 10.3389/fspas.2019.00053
Planck, SPT, and ACT surveys have clearly demonstrated that Cosmic Microwave Background (CMB) experiments, while optimized for cosmological measurements, have made important contributions to the field of extragalactic astrophysics in the last decade. Future $\mathrm{CMB}$ experiments have the potential to make even greater contributions. One example is the detection of high-z galaxies with extreme gravitational amplifications. The combination of flux boosting and of stretching of the images has allowed the investigation of the structure of galaxies at $z \simeq 3$ with the astounding spatial resolution of about $60 \mathrm{pc}$. Another example is the detection of proto-clusters of dusty galaxies at high $z$ when they may not yet possess the hot intergalactic medium allowing their detection in X-rays or via the Sunyaev-Zeldovich effect. Next generation CMB experiments, like PICO, CORE, CMB-Bharat from space and Simons Observatory and CMB-S4 from the ground, will discover several thousands of strongly lensed galaxies out to $z \sim 6$ or more and of galaxy proto-clusters caught in the phase when their member galaxies where forming the bulk of their stars. They will also detect tens of thousands of local dusty galaxies and thousands of radio sources at least up to $z \simeq 5$. Moreover they will measure the polarized emission of thousands of radio sources and of dusty galaxies at $\mathrm{mm} / \mathrm{sub}-\mathrm{mm}$ wavelengths.

Keywords: cosmic microwave background, galaxy surveys, radio sources, strong lensing, sub-millimeter galaxies, proto-clusters

\section{INTRODUCTION}

WMAP and even more Planck have already provided an exciting foretaste of the potential of space-borne Cosmic Microwave Background (CMB) experiments for extragalactic astrophysics. Next generation experiments with telescope sizes similar to Planck's, like the Cosmic Origins Explorer (CORE; Delabrouille et al., 2018), the Probe of Inflation and Cosmic Origins (PICO; Hanany et al., 2019) or CMB Bharat ${ }^{1}$ can do much better. This is because, for state-of-the-art instruments with sensitivity at fundamental limits (as Planck also was) the detection limit is not

\footnotetext{
${ }^{1}$ http://cmb-bharat.in/
} 
set by instrumental noise but by fluctuations of astrophysical foregrounds (confusion noise), whose amplitude, at the frequencies and angular scales of interest, is roughly proportional to the beam solid angle (see Figure 3 of De Zotti et al., 2015). Planck did not work at the diffraction limit while next generation experiments will. The improvement in angular resolution will be substantial. For example, at $545 \mathrm{GHz}(550 \mu \mathrm{m})$ the Planck beam has an effective full-width at half maximum FWHM $=4.83^{\prime}$ (Planck Collaboration III, 2018) while the diffraction limit for its $1.5 \mathrm{~m}$ telescope is $1.5^{\prime}$. Improving the resolution to the diffraction limit decreases the detection limit at this frequency by about one order of magnitude.

The De Zotti et al. (2015) results on the impact of angular resolution on the detection limits, based on simulations exploiting the Planck sky model (Delabrouille et al., 2013), have been improved using real data. To this end we degraded the publicly available South Pole Telescope (SPT) maps of $2540 \mathrm{deg}^{2}$ at 95, 150, and $220 \mathrm{GHz}$ (Chown et al., 2018), with resolutions of approximately $1.7^{\prime}, 1.2^{\prime}$, and $1.0^{\prime}$, respectively, to the PICO resolution (FWHM $=9.5^{\prime}, 6.2^{\prime}$, and 3.6', respectively) and applied to the degraded maps the Mexican Hat Wavelet 2 (MHW2) source detections algorithm (GonzálezNuevo et al., 2006; López-Caniego et al., 2006), also used to build the Second Planck Catalog of Compact Sources (PCCS2; Planck Collaboration XXVI, 2016). The results at 150 and $220 \mathrm{GHz}$ are illustrated by Figure 1. The completeness limits for the degraded maps were obtained by comparison with the SPT counts. We found $90 \%$ completeness down to 95,70 , and $55 \mathrm{mJy}$ at 95, 150, and $220 \mathrm{GHz}$, respectively. For comparison, the PCCS2 90\% completeness limits ${ }^{2}$ in the "extragalactic zone" are 269, 177, and $142 \mathrm{mJy}$, respectively (Planck Collaboration III, 2018). The PICO detection limits at the other frequencies were obtained by means of analytical extrapolations of results obtained from simulations done for the CORE project (De Zotti et al., 2018). These extrapolations were found to be consistent with the determinations based on degraded SPT maps and with the PCCS2 90\% completeness limits in the "extragalactic zone." Similar detection limits hold for CORE and CMB Bharat.

Fluctuations (hence detection limits) at few arcmin resolution are dominated by compact sources too faint to be detected individually. Such source confusion can be reliably determined from the power spectra of the Cosmic Infrared Background (CIB), measured by Planck (Planck Collaboration XVIII, 2011; Planck Collaboration XXX, 2014) and by Herschel (Viero et al., 2013). On larger angular scales, however, fluctuations of diffuse emissions (Galactic or CMB) must also be taken into account.

The potential of ground based CMB experiments has been demonstrated by the SPT (Mocanu et al., 2013) and by the Atacama Cosmology Telescope (ACT; Marsden et al., 2014) surveys. With telescopes of the 6-10 m class, these experiments reach arcmin angular resolution at $\mathrm{mm}$ wavelengths, with sensitivity at fundamental limits. Next generation experiments, like CMB-S4 (Abazajian et al., 2016) and the Simons Observatory

\footnotetext{
${ }^{2}$ Effective FWHM $=9.68^{\prime}, 7.30^{\prime}$, and $5.02^{\prime}$ at 100,143 , and $217 \mathrm{GHz}$, respectively; FWHMs of the Gaussians whose solid angle is equivalent to that of the effective beams.
}

(Ade et al., 2019) will extend the coverage to at least $40 \%$ of the sky.

Space-borne experiments like PICO will cover a very broad frequency range, from $\simeq 20$ to $\simeq 800 \mathrm{GHz}$, while observations from the ground are only possible, in atmospheric windows, up to $\simeq 300 \mathrm{GHz}$. The extragalactic sky in the frequency range of CMB experiments is dominated by two point source populations: blazars (Flat-Spectrum Radio Quasars and BL Lacertae-type sources) and dusty star-forming galaxies. Blazars are powerful radio-loud active galactic nuclei (AGNs) whose relativistic jets are closely aligned with the line of sight. They overbear the number counts at wavelengths longer than about $1 \mathrm{~mm}$ while dusty galaxies prevail at shorter wavelengths. This is illustrated by Figure 2, where the spectral energy distributions (SEDs) of a $z \simeq 2$ blazar and of two dusty galaxies are compared with the estimated detection limits of the PICO project (Hanany et al., 2019) and with the completeness limits of the SPT surveys (Mocanu et al., 2013), extrapolated on one side to $40 \mathrm{GHz}$ and on the other side to $270 \mathrm{GHz}$ to cover the frequency range of CMB-S4. The 90\% completeness limits of the PCCS2 in the "extragalactic zone" are also shown for comparison.

In this paper we will discuss the expected outcome of planned next generation $\mathrm{CMB}$ experiments with regard to strongly gravitationally lensed high- $z$ dusty galaxies (section 2), galaxy proto-clusters (section 3 ) and radio sources (section 4). In section 5 we deal with counts of the various classes of extragalactic sources in polarization. The main conclusions are summarized in section 6

We adopt a flat $\Lambda \mathrm{CDM}$ cosmology with the latest values of the parameters derived from Planck CMB power spectra: $H_{0}=67.4 \mathrm{~km} \mathrm{~s}^{-1} \mathrm{Mpc}^{-1}$ and $\Omega_{m}=0.315$ (Planck Collaboration VI, 2018).

\section{DUSTY GALAXIES}

\subsection{Strongly-Lensed Galaxies}

Next generation CMB experiments will conduct a census of the brightest sub-mm galaxies in the Universe. Planck, SPT and ACT have already offered an exciting foretaste of that. Follow-up CO spectroscopy and multi-frequency photometry of 11 "Planck's dusty GEMS” (Gravitationally Enhanced subMillimetre Sources; Cañameras et al., 2015) have shown that they are at $z=2.2-3.6$. Their apparent (uncorrected for gravitational amplification) farIR luminosities are up to $3 \times 10^{14} \mathrm{~L}_{\odot}$, making them among the brightest sources in the Universe.

Herschel extragalactic surveys yielded a surface density of $\simeq 0.16 \mathrm{deg}^{-2}$ for $S_{500 \mu \mathrm{m}} \geq 100 \mathrm{mJy}$ (Negrello et al., 2017a). A space-borne $1.5 \mathrm{~m}$ telescope will reach a slightly fainter flux density limit over the full sky (excluding the region around the Galactic plane), thus achieving the detection of several thousands of strongly lensed galaxies (upper left-hand panel of Figure 3). The number of detections decreases rapidly for smaller telescope sizes; a telescope substantially smaller than $1 \mathrm{~m}$ cannot do any better than Planck. A $2 \mathrm{~m}$ telescope would increase the number of strongly lensed detections by a factor $\simeq 2.5$. However their selection would require additional information to distinguish them from unlensed high- $z$ galaxies dominating the counts at the 


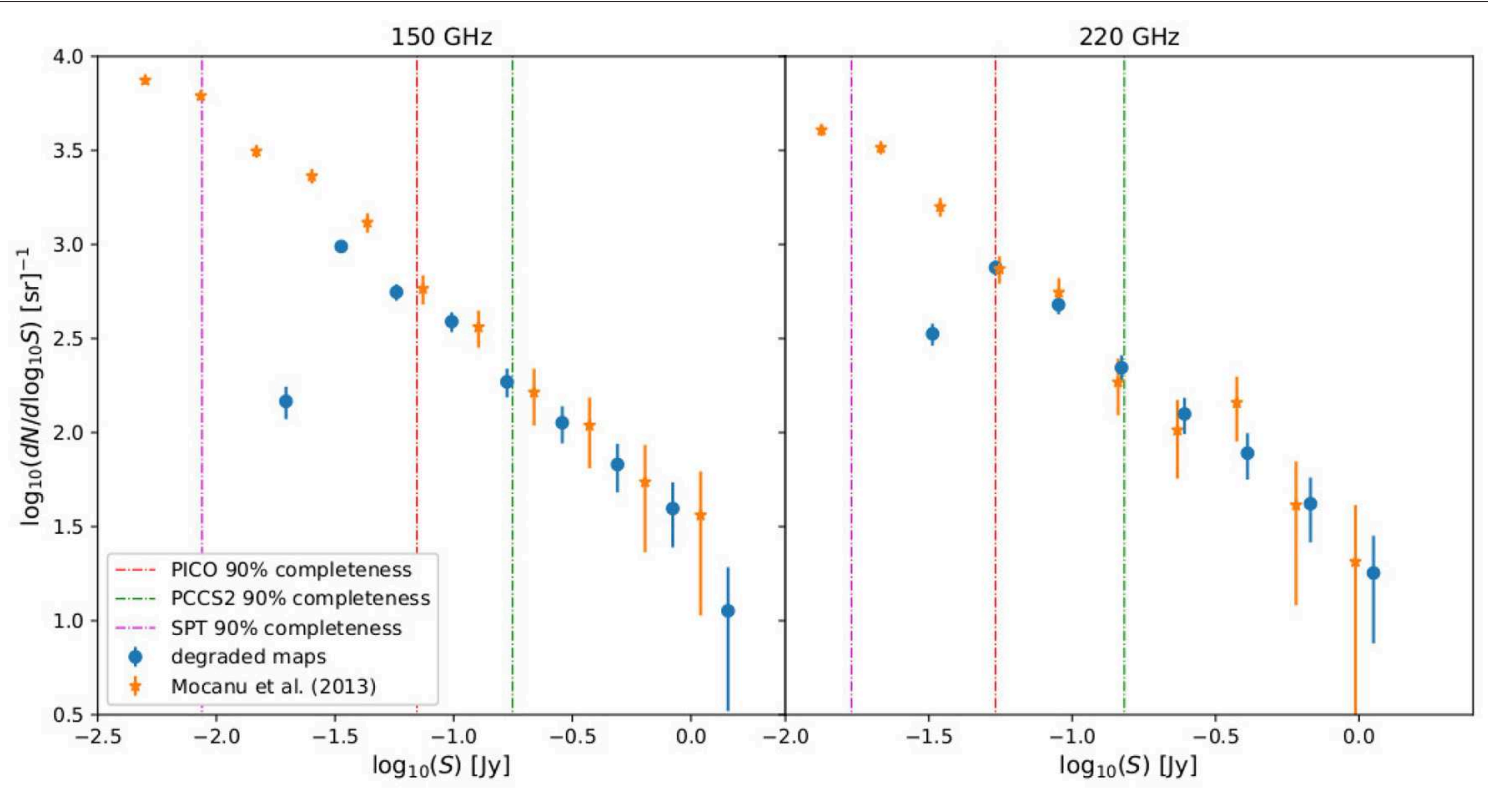

FIGURE 1 | Effect of angular resolution on the confusion limit. The filled blue circles show the differential counts of sources on SPT maps degraded to the PICO resolution at $150 \mathrm{GHz}\left(\mathrm{FWHM}=6.2^{\prime}\right.$; left panel) and $220 \mathrm{GHz}\left(\mathrm{FWHM}=3.6^{\prime}\right.$; right panel) compared with the SPT counts of Mocanu et al. (2013, orange stars). The vertical dot-dashed lines correspond, from left to right, to the $90 \%$ completeness limits at the full SPT resolution, at the PICO resolution and at the Planck resolution.
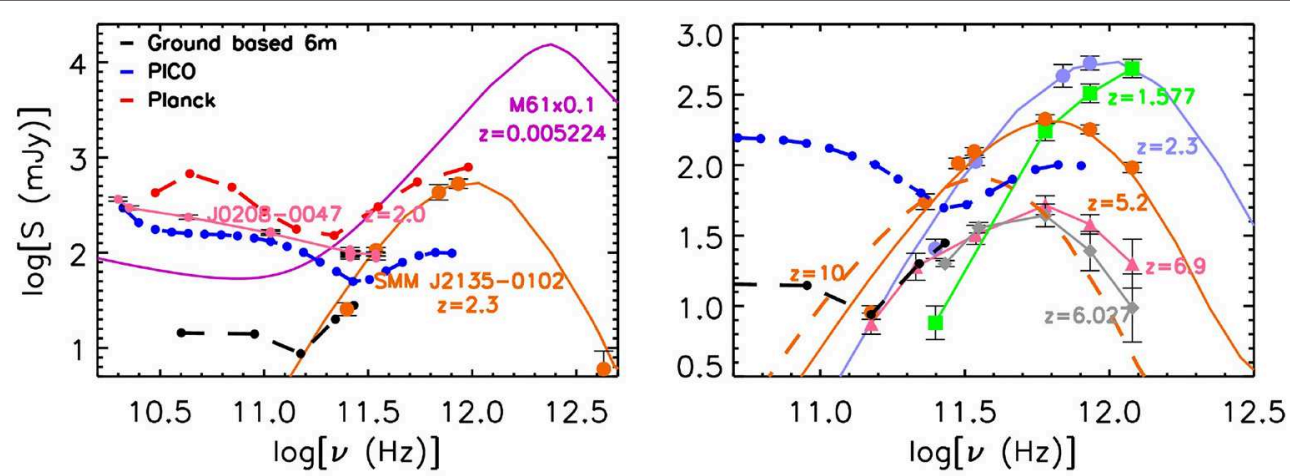

FIGURE 2 | Spectral energy distributions of extragalactic sources in the frequency range of CMB experiments, compared with the estimated detection limits of the PICO project and with the completeness limits of the SPT surveys extrapolated to 40 and to $270 \mathrm{GHz}$, to cover the range of the CMB-S4 project. The PCCS2 $90 \%$ completeness limits in the "extragalactic zone" are also shown for comparison (in the left panel only). At wavelengths shorter than a few mm the dominant extragalactic population are dusty star-forming galaxies. At longer wavelengths radio sources, mostly blazars, take over. The spectral energy distributions (SEDs) of blazars are characterized by a relatively flat continuum spectrum exemplified by that of J0208-0047 at $z \simeq 2.0$ (flat solid orange line). Dusty star-forming galaxies are a mixture of local and high-z strongly gravitationally lensed objects. Local galaxies are, by far, the brightest extragalactic sources at sub-mm wavelengths. Their SEDs are exemplified by that of the dusty star-forming galaxy M 61, scaled down by a factor of 10. Some examples of SEDs of strongly lensed galaxies are shown: SDP 9 ( $z=1.577$; Negrello et al., 2010), SMMJ2135-0102 ( $z=2.3259$; Swinbank et al., 2010), HLS J091828.6+514223 ( $z=5.2 ;$ Combes et al., 2012), HATLAS J090045.4+004125 ( $z=6.027$; Zavala et al., 2018), and SPT-S J031132-5823.4 ( $z=6.9$; Strandet et al., 2017). The dashed orange SED represents that of HLS J091828.6+514223 scaled to $z=10$ to show that galaxies like it are visible up to very high redshifts both by ground-based and by space-borne next generation CMB experiments.

corresponding flux density limit. Efficient methods to separate lensed and unlensed galaxies have been presented by GonzálezNuevo et al. (2012) and González-Nuevo et al. (2019).

Ground-based instruments typically have higher resolution at the longer wavelengths, and thus select a higher redshift population. A straightforward extrapolation of results from analyses in the first (Vieira et al., 2010) and second (Mocanu et al., 2013) SPT catalog papers imply that the SPT has detected hundreds of strongly lensed galaxies over the full survey area (lower left-hand panel of Figure 3). Their redshift distribution (Vieira et al., 2013; Weiß et al., 2013) is broader than found for Herschel surveys (Negrello et al., 2017a) and the mean redshift, $\bar{z}=3.5$ (Weiß et al., 2013) is higher (lower right-hand panel of Figure 3). The maximum spectroscopically measured redshift, $z=6.9$ (Strandet et al., 2017), shows that this survey has reached the epoch of reionization. Additional samples of strongly lensed 

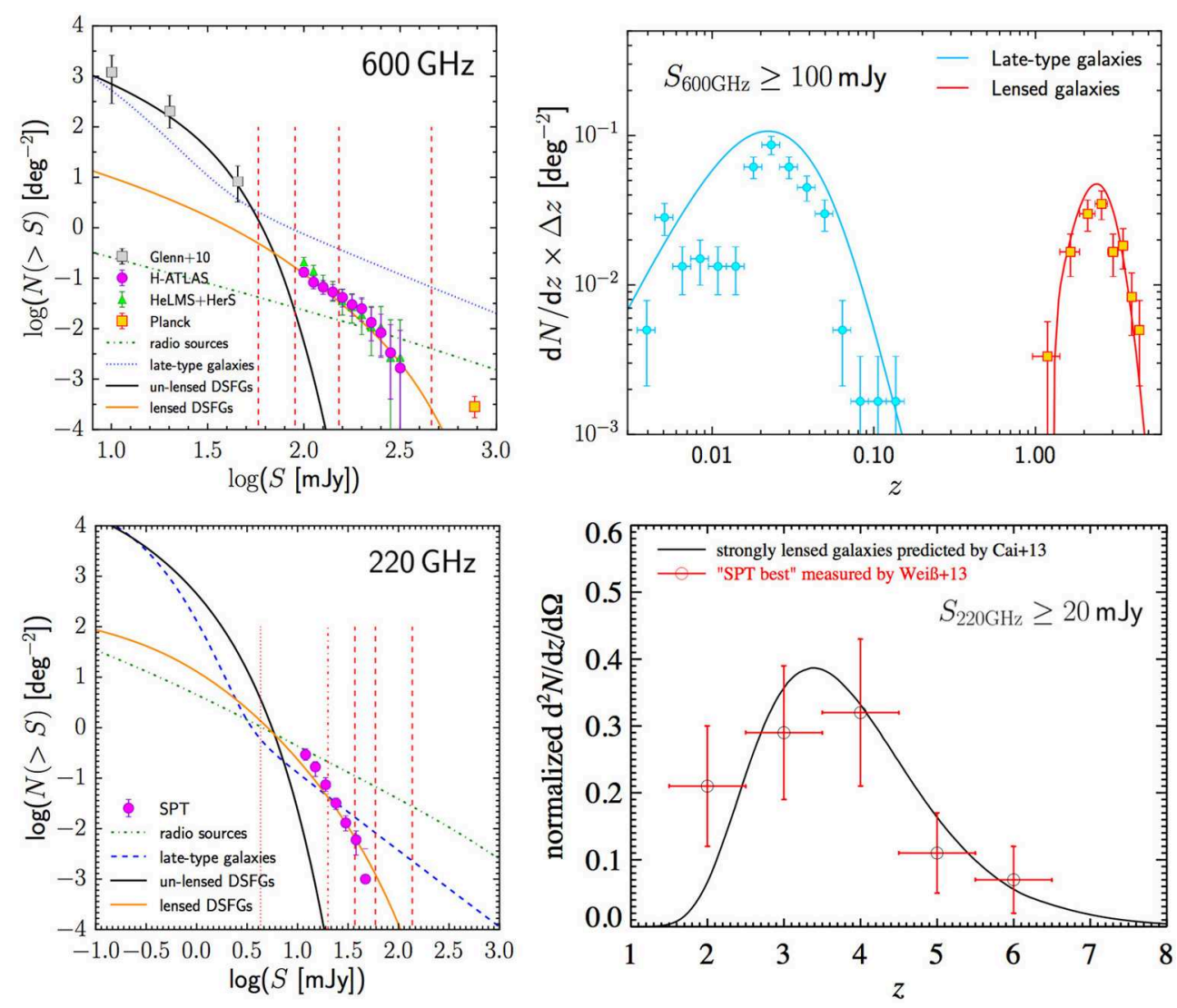

FIGURE 3 | (Upper left panel) Integral counts of strongly lensed galaxies from Herschel surveys at $500 \mu \mathrm{m}$ (600 GHz; green and magenta data points from Negrello et al., 2017a), compared with the predictions of the Cai et al. (2013) model (solid orange line). The yellow square on the bottom-right corner is our own estimate of the counts of strongly lensed galaxies detected by Planck. The counts of unlensed proto-spheroidal galaxies (data points from Glenn et al., 2010 , black squares; model by Cai et al., 2013, solid black line), of radio sources (dotted green line, model by Tucci et al., 2011) and of normal and starburst late-type galaxies (dotted blue line, model by Cai et al., 2013) are also shown for comparison. The vertical dashed orange lines show, from right to left, the $90 \%$ completeness limit of the PCCS2 and the $5 \sigma$ detection limits for space-borne CMB experiments with 1, 1.5 and $2 \mathrm{~m}$ telescopes working at the diffraction limit. This panel shows a modified version of Figure 8 of Negrello et al. (2017a). (Upper right panel) Redshift distribution of galaxies brighter than $100 \mathrm{mJy}$ at $500 \mu \mathrm{m}$, derived from the full H-ATLAS catalog (data points with Poisson errors) compared with the predictions of the Cai et al. (2013) model (solid lines). There is a clear bimodality. On one side we have nearby late-type galaxies, almost all at $z \leq 0.06$, and hence easily recognizable in optical/near-infrared catalogs. On the other side we have dust enshrouded, hence optically very faint, gravitationally lensed galaxies at $z \geq 1$ and up to $z>4$. (Lower left panel) Integral counts of strongly lensed galaxies from the SPT survey at $220 \mathrm{GH} z$ ( $1.4 \mathrm{~mm}$; magenta data points; Vieira et al., 2010) compared with the prediction of the Cai et al. (2013, orange solid line) model. Counts of radio sources and of late-type galaxies are from the same models as in the upper panel. The vertical lines show, from right to left, the $5 \sigma$ detection limits for diffraction limited $1,1.5$, and $2 \mathrm{~m}$ telescopes, the completeness limit of the SPT survey (Mocanu et al., 2013) and the $5 \sigma$ confusion limit for a $6 \mathrm{~m}$ telescope. (Lower right panel) Estimated redshift distribution of strongly lensed galaxies with $S_{220 ~ G H z}>20$ mJy (Weiß et al., 2013) compared with the prediction of the Cai et al. (2013) model.

galaxies, over a similar redshift range have been provided by the ACT surveys (Marsden et al., 2014; Su et al., 2017).

Next-generation ground-based experiments, extending the sky coverage up to $40 \%$ of the sky or more, will detect thousands of strongly lensed galaxies if their performances will be similar to those of the SPT. If these experiments will be able to reach the confusion limit of a $6 \mathrm{~m}$ telescope operating at the diffraction limit (4-5 mJy), the number of strongly-lensed detections will increase to tens of thousands.

The right-hand panel of Figure 2 shows that both groundbased and space-borne experiments will detect strongly lensed galaxies up to high redshifts. For example objects like the strongly lensed galaxy HLS J091828.6+514223 at $z=5.2$ would be detectable up to $z$ of at least 10 (dashed SED in
Figure 2). Ground-based and space-borne experiments cover complementary redshift ranges. The former are more efficient at $z \gtrsim 2$, the latter at lower $z$.

These surveys will also probe a large fraction of the entire Hubble volume for the most intense hyper-luminous starbursts, testing whether there are physical limits to the star-formation rates (SFRs) of galaxies (e.g., Crocker et al., 2018).

\subsection{Comparison With the Selection of Strongly Lensed Galaxies in Other Wavebands}

Herschel surveys have demonstrated that at $500 \mu \mathrm{m}(600 \mathrm{GHz})$ strongly lensed galaxies with $S_{500 \mu \mathrm{m}} \geq 100 \mathrm{mJy}$, close to the 


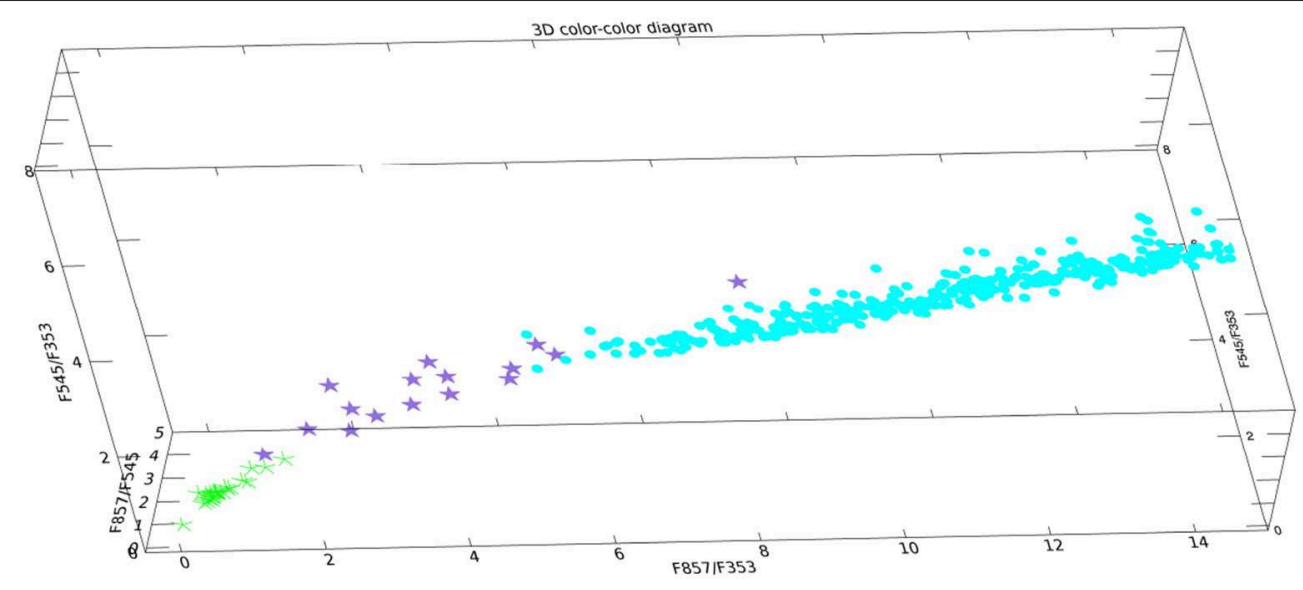

FIGURE 4 | 3D color-color plot showing the colors of strongly-lensed galaxies detected by Planck (purple stars) compared to those of local galaxies (light blue symbols) and of radio sources (green symbols). The distribution of local galaxies extends to the blue far beyond the chosen boundaries of the figure. Strongly lensed galaxies populate a region intermediate between those of local galaxies and radio sources and well distinct from both.

detection limit of next generation space-borne CMB experiments with telescopes of the $\simeq 1.5 \mathrm{~m}$ class operating at the diffraction limit, amount to $\simeq 25 \%$ of the total counts (Nayyeri et al., 2016; Negrello et al., 2017a, cf. upper left panel of Figure 3). A similar fraction was found at the completeness limit $(\simeq 20 \mathrm{mJy})$ of the SPT survey at $220 \mathrm{GHz}$ (Mocanu et al., 2013). Such high fractions are a direct consequence of the extreme steepness of (sub-) $\mathrm{mm}$ counts of extragalactic sources due to the unique combination of steep cosmological evolution with a strongly negative Kcorrection $^{3}$. They are an exclusive property of (sub-)mm surveys: searches in other wavebands have yielded fractions of $\simeq 0.1 \%$ (York et al., 2005; Jackson, 2008; Treu, 2010).

Not only the strongly-lensed fractions are high, but also the selection is easy done, with close to $100 \%$ efficiency (Negrello et al., 2007, 2010), while it remains a significant challenge in other wavebands (Jacobs et al., 2019). The other extragalactic sources brighter than the detection limits of next generation CMB experiments are either $z \lesssim 0.1$ galaxies or radio sources (mainly blazars), that can be identified using existing all-sky optical or radio catalogs.

In the case of space-borne experiments, the selection can be done directly on survey data. As illustrated by the upper righthand panel of Figure 3, the redshift distribution of dusty galaxies detected by these experiments is highly bimodal. On one side we have nearby late-type galaxies, almost all at $z \leq 0.06$; on the other side we have dust enshrouded, hence optically very faint, gravitationally lensed galaxies at $z \geq 1$ and up to $z>4$. This strong difference in redshift translates in a clear difference in sub-mm colors, high- $z$ galaxies being much redder than the local ones; radio sources have still redder colors (see Figure 4). This is specific to searches with these experiments. Selections in

\footnotetext{
${ }^{3}$ The steep increase with increasing frequency of the dust emission spectrum at (sub-)mm wavelengths $\left(L_{v} \propto v^{\alpha}\right.$ with $\left.\alpha \simeq 3.5-4\right)$ translates into a fast increase of the observed flux density with increasing redshift (negative K-correction). Such increase may compensate and even exceed the decrease due to increasing distance (Franceschini et al., 1991; Blain and Longair, 1993).
}

other wavebands need spectroscopy or other ancillary data. This is true also for ground-based surveys which provide photometry in the Rayleigh-Jeans region where colors are largely redshiftindependent. On the other hand, the discrimination of strongly lensed galaxies will be greatly eased by the wealth of multiwavelength data over the entire sky that will be available in the coming decade.

In addition to the much more efficient selection, other critical advantages of $\mathrm{CMB}$ experiments over other facilities that will generate large gravitational lens catalogs (e.g., Euclid, Gaia, SKA; Serjeant, 2017) are:

- compared to optical/near-IR surveys, they detect earlier (higher redshift) phases of galaxy evolution, characterized by intense, dust-enshrouded star-formation activity;

- the photometry of detected galaxies will be only very weakly contaminated by the foreground lens, even in the case of close alignment along the line-of-sight (see Figure 5); while the lensed galaxies are heavily dust enshrouded, hence bright at sub-mm wavelengths but faint in the optical, the foreground lenses are mostly massive early-type galaxies in passive evolution, hence optically bright but almost invisible in the sub-mm;

- the all-sky coverage maximizes the detections of the rare brightest sources, with the most extreme magnifications, optimally suited for follow-up, as demonstrated by Planck: the magnification factors of "Planck's dusty GEMS" are estimated to be of up to 50 (Cañameras et al., 2015);

- the $\mathrm{mm} / \mathrm{sub}-\mathrm{mm}$ selection, with its strongly negative $\mathrm{K}$ correction, allows us to extend the detection of sources and lenses to much higher redshifts than in any other waveband.

\subsection{Astrophysics and Cosmography With Strong Lensing}

The extreme magnifications of dusty galaxies detected by CMB experiments makes them trivially easy targets for ALMA, 


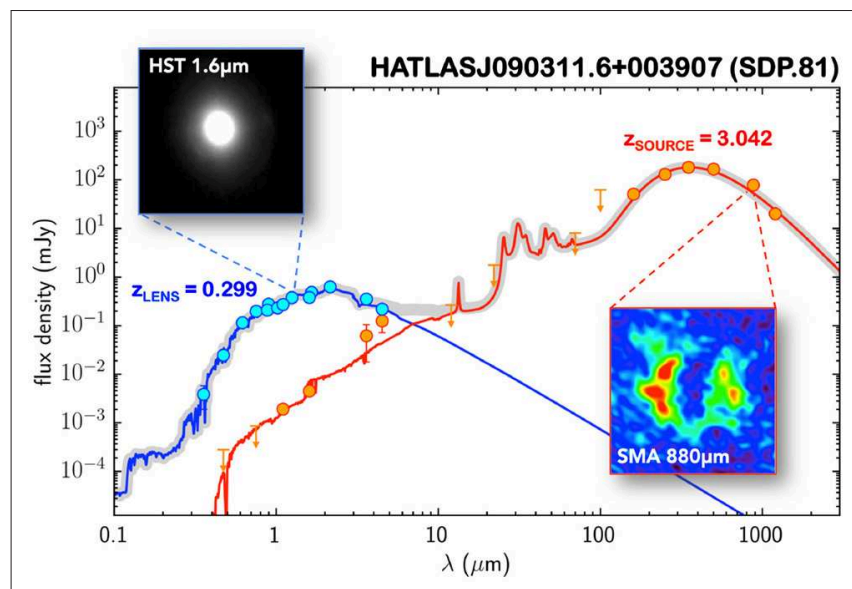

FIGURE 5 | SEDs of the strongly lensed galaxy SDP 81 at 3.042 detected by the Herschel Astrophysical Terahertz Large Area Survey (H-ATLAS; Eales et al., 2010) and imaged with the Sub-Millimeter Array (SMA) at $880 \mu \mathrm{m}$ (Negrello et al., 2010) and of the foreground lens at $z=0.299$ imaged with the Wide Field Camera 3 (WFC3) on the Hubble Space Telescope (HST; Negrello et al., 2014). As is generally the case, the lens is a spheroidal galaxy in passive evolution, hence very faint at sub-mm wavelengths; indeed it is essentially invisible in the SMA image. The lensed source is dust-enshrouded, hence bright in the sub-mm but very faint in the optical and essentially invisible in the WFC3/HST image.

NOEMA, SMA etc., and the foreground lenses will almost certainly be detectable by large optical telescopes and in, e.g., Euclid imaging. Follow-up observations will allow us to address major astrophysical issues (e.g., Treu, 2010).

$\mathrm{CMB}$ experiments with arcmin resolution both from the ground and from space will drive a real breakthrough in the study of early evolutionary phases of galaxies, paving the way to answer major, still open problems like: which are the physical mechanisms shaping the galaxy properties (Silk and Mamon, 2012; Somerville and Davé, 2015): in situ processes? interactions? mergers? cold flows? How feedback processes work? To settle these issues we need direct information on the structure and the dynamics of high- $z$ galaxies. But these are compact, with typical sizes of 1-2 kpc (e.g., Enia et al., 2018; Fujimoto et al., 2018), corresponding to angular sizes of $0.1-0.2 \operatorname{arcsec}$ at $z=2-3$. Thus they are hardly resolved even by ALMA and by the HST. If they are resolved, high enough signal-to-noise ratios per resolution element are achieved only for the brightest galaxies, not representative of the general population.

Strong gravitational lensing provides a solution. CMB surveys will detect the brightest (sub-)mm strongly lensed galaxies in the sky, with extreme magnifications, up to several tens (Cañameras et al., 2015). Since lensing conserves surface brightness, the effective angular size is stretched by an average factor $\mu^{1 / 2}$, substantially increasing the resolving power. A spectacular example are ALMA 0.1 arcsec resolution observations of PLCK G244.8+54.9 at $z \simeq 3.0$ with $\mu \simeq 30$ (Cañameras et al., 2017a) which reached the astounding spatial resolution of $\simeq 60 \mathrm{pc}$, substantially smaller than the size of Galactic giant molecular clouds.
Cañameras et al. (2017a) also obtained CO spectroscopy with an uncertainty of $40-50 \mathrm{~km} / \mathrm{s}$. This spectral resolution makes possible a direct investigation of massive outflows driven by AGN feedback at high $z$, with predicted velocities of $\sim 1000 \mathrm{~km} \mathrm{~s}^{-1}$ (King and Pounds, 2015). AGN driven outflows are a key ingredient of current galaxy evolution models since they provide the most plausible explanation for the deviation of the galaxy stellar mass function from the halo mass function at large masses, i.e., for the low star-formation efficiency in massive halos: only less than $10 \%$ of baryons initially present in such halos are used to form stars. However, until very recently, with few exceptions (Cicone et al., 2015), high- $z$ outflows were detected only in ionized gas (Cresci and Maiolino, 2018). Thus information on the effect of feedback on the direct fuel of star formation, molecular gas, has been largely missing during the epoch of the most active cosmic star formation.

Due to the weakness of spectral signatures of molecular outflows, observational progress has been difficult. Gravitational lensing allows us to overcome these difficulties. Spilker et al. (2018) were able to detect, by means of ALMA spectroscopy, a fast $(800 \mathrm{~km} / \mathrm{s})$ molecular outflow in a strongly lensed galaxy at $z=5.3$, discovered by the SPT survey. The outflow carries mass at a rate close to the SFR, thus removing a large fraction of the gas available for star-formation. Cañameras et al. (2018) detected a molecular wind signature in the strongly lensed galaxy PLCK G165.7+49.0, discovered by Planck, at $z=2.236$, with magnification factors between 20 and 50 in most of the source. Strongly lensed galaxies detected by CMB experiments will be obvious targets for the next generation Very Large Array (ngVLA) that will provide accurate estimates of the molecular outflow masses and mass loss rates up to $z \simeq 4$ (Spilker and Nyland, 2018). The proposed NASA flagship Origins Space Telescope (OST; Leisawitz et al., 2018; Bonato et al., 2019a) would detect molecular, neutral, and warm ionized phases of outflows up to higher redshifts, although with a spatial resolution lower than ngVLA. AGN-driven outflows also produce a bubble of hot gas, potentially detectable via its Sunyaev-Zeldovich effect (SZE; Natarajan and Sigurdsson, 1999; Platania et al., 2002). The first detection of the SZE from a hyperluminous quasar at $z=1.71$, obtained by means of ALMA observations, was reported by Lacy et al. (2019) who, from these measurements, derived constraints on the energetics of the wind.

The high redshifts of magnified galaxies imply high redshifts of foreground lenses. Optical follow-up allows us to investigate the total (visible and dark) mass of the lensing galaxies, their density profiles, dark matter sub-structures at higher redshifts than in the case of optical selection. For example, the spectroscopic redshift of the foreground deflector of the strongly lensed galaxy PLCKG244.8+54.9 ( $z=3.005)$, measured by Cañameras et al. (2017b), was found to be exceptionally high, $z=1.525$. This illustrates the power of the (sub-)mm selection of strongly lensed galaxies to push the study of dark matter and baryon assembly up to high redshifts.

The samples of thousands of strongly lensed galaxies provided by $\mathrm{CMB}$ experiments will also be a powerful tool to measure cosmological parameters (Treu, 2010; Eales, 2015). The lens equation contains ratios of angular diameter distances. Hence 
observables of lensed systems (redshifts of the source and of the lens, Einstein radius, velocity dispersion of the lens) can be used to estimate $\Omega_{m}, \Omega_{\Lambda}$ and the parameters of the dark energy equation of state. These determinations will not be as precise as those obtained from $\mathrm{CMB}$ data, but will provide valuable independent tests of the cosmological model, will allow to look for unrecognized selection effects and to break degeneracies in the interpretation of $\mathrm{CMB}$ data. Also, the strong lensing optical depth depends on the abundance and redshift distributions of potential wells acting as deflectors, hence on density parameters, on the dark energy equation of state and its evolution and on the amplitude, $\sigma_{8}$, of the primordial perturbation spectrum. Distortions of Einstein rings or giant arcs could offer a direct method to detect sub-halos, i.e., to constrain warm dark matter (Li et al., 2016). Lenses at high redshift are particularly valuable to determine the statistics of perturbations along the line of sight (Treu, 2010).

\subsection{Local Dusty Galaxies}

Herschel surveys have shown that the surface density of local dusty galaxies brighter than $S_{500 \mu \mathrm{m}}=100 \mathrm{mJy}$ is $\simeq 1 \mathrm{deg}^{-2}$ and that their redshift distribution peaks at $z=0.02-0.03$ (upper right panel of Figure 3; Negrello et al., 2017a). A survey with a space borne $\simeq 1.5 \mathrm{~m}$ telescope will detect tens of thousands of them. By the time when next generation CMB experiments will fly several wide-angle redshift and photometric surveys will be available, providing distance information for the majority, if not all of them (De Zotti et al., 2018). Combining these data with available or forthcoming data in different wavebands (radio, IRAS, AKARI, WISE, Euclid, GALEX, ROSAT, eROSITA...) it will be possible to determine, for each galaxy type and as a function of stellar mass, the distribution of dust temperatures and masses, the SFR function, the relationship between star formation and nuclear activity, the contributions of newborn and evolved stars to dust heating, and more. The sample of local galaxies will also be large enough for clustering studies, i.e., to relate the properties of galaxies to the underlying dark matter field and to the properties of their dark matter haloes, as well as to investigate the link between galaxies of different types and their environments.

The surface density of local galaxies at the SPT completeness limit at $220 \mathrm{GHz}(20 \mathrm{mJy})$ is of $\simeq 0.05 \mathrm{deg}^{-2}$ (lower left panel of Figure 3), i.e., a factor of $\simeq 20$ lower than achieved by a spaceborne $1.5 \mathrm{~m}$ telescope at $600 \mathrm{GHz}$. However, as shown by the SED of M 61 scaled down by a factor of 10 (Figure 2), ground based surveys will detect, for the nearest low- $z$ galaxies, also the radio emission powered by star formation. At $\mathrm{mm}$ wavelength such radio emission is expected to be dominated by free-free emission (Galvin et al., 2018) which is an excellent measure of the instantaneous SFR since it scales with the ionizing luminosity and is unaffected by extinction. Its measurement will allow us to recalibrate the relation between dust emission (which may be contributed in part by dust heating from old stars) and SFR. According to the calculations by Mancuso et al. (2015), the surface density of star-forming galaxies detected via their radio emission at $95 \mathrm{GHz}$ by SPT-like experiments, i.e., brighter than $S_{95 \mathrm{GHz}} \simeq 10 \mathrm{mJy}$, is of $\simeq 12 \mathrm{sr}^{-1}$. We caution, however, that the measurement of the free-free emission requires the separation of the non-thermal (synchrotron) and dust contributions. This is very difficult to do accurately, especially because of uncertainties on the synchrotron spectrum (Aravena et al., 2013).

Another open issue is the connection between nuclear radio activity and star formation. Planck has detected evidence of cold dust associated to a handful of nearby radio sources, based on their rising spectra at $\mathrm{mm}$ wavelengths (Planck Collaboration XLV, 2016). The much deeper surveys carried out by nextgeneration experiments can push the investigation to much more distant objects.

\section{EARLY PHASES OF CLUSTER EVOLUTION}

Understanding the full evolutionary history of present-day galaxy clusters is of fundamental importance for the observational validation of the formation history of the most massive dark matter halos, a crucial test of models for structure formation, and for investigating the impact of environment on the formation and evolution of galaxies. Because of their deep potential wells, clusters may preserve fingerprints of the physical processes responsible for triggering and suppression of star formation and black hole activity. Also, clusters of galaxies have historically been powerful probes of cosmological parameters (e.g., Bocquet et al., 2018).

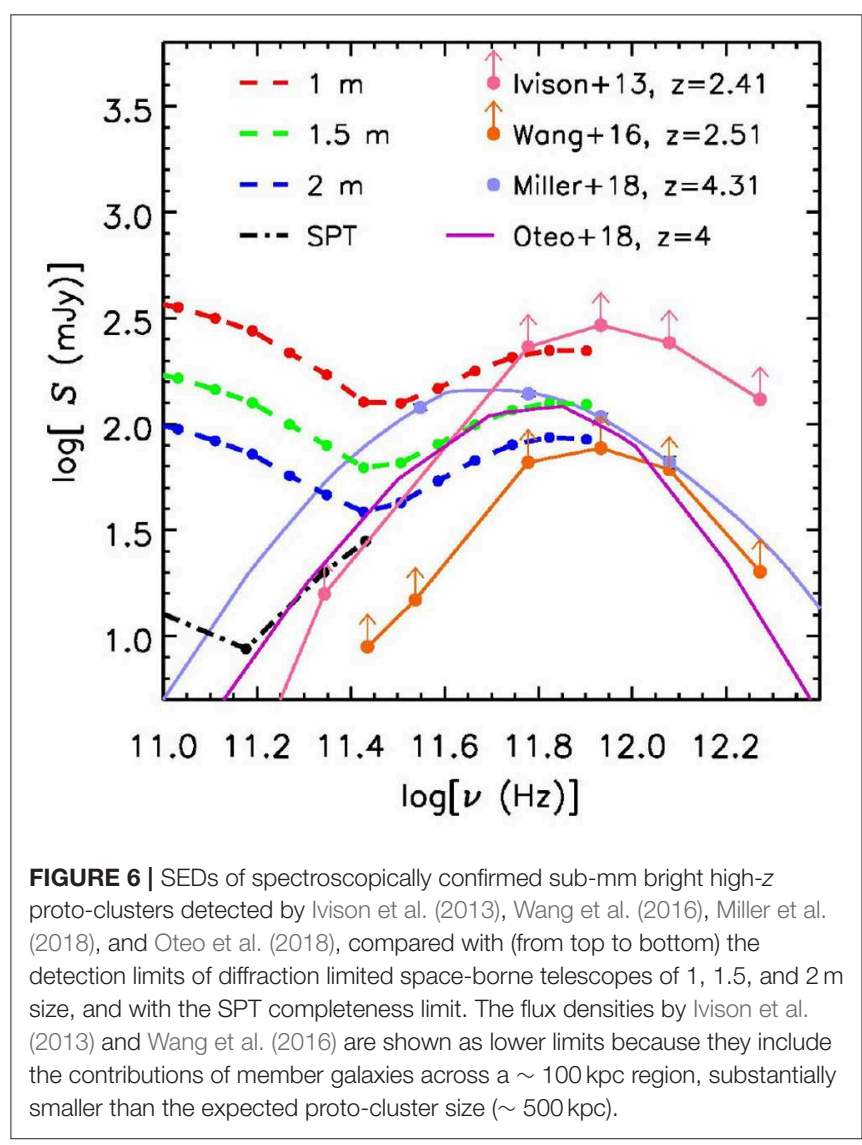



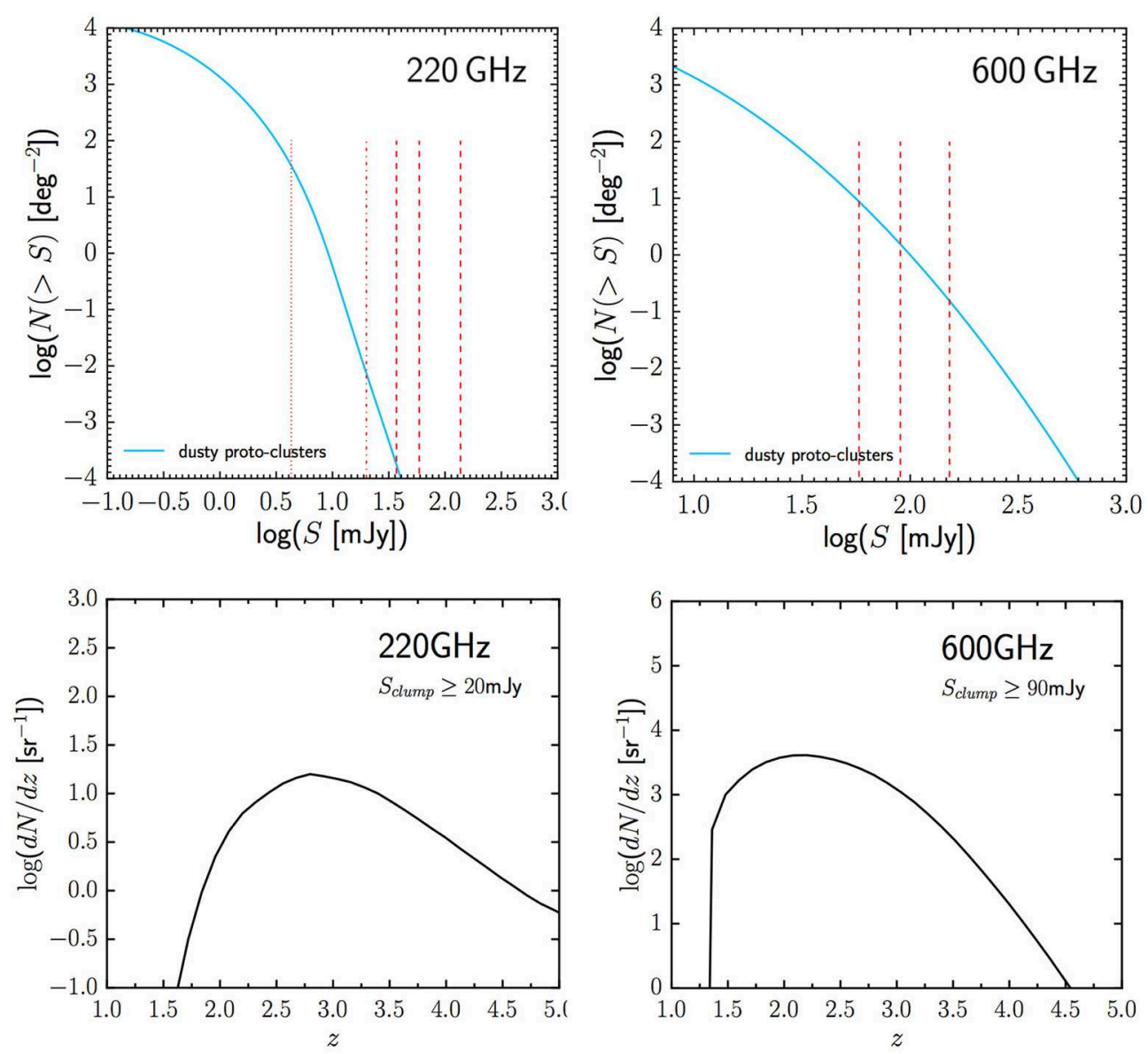

FIGURE 7 | Integral counts and redshift distributions of proto-clusters at $220 \mathrm{GHz}$ and $600 \mathrm{GHz}$ ( $1.4 \mathrm{~mm}$ and $500 \mu \mathrm{m}$, respectively) predicted by Negrello et al. (2017b). The vertical lines in the upper left panel show, from left to right, the $5 \sigma$ confusion limit for a $6 \mathrm{~m}$ telescope, the SPT completeness limit and the $5 \sigma$ detection limits for 2, 1.5 and $1 \mathrm{~m}$ telescopes operating at the diffraction limit. Only the three latter limits, at $600 \mathrm{GHz}$, are shown in the upper right-hand panel. The lower panels show the redshift distributions at the SPT completeness limit at $220 \mathrm{GHz}$ ( $20 \mathrm{mJy}$, left) and at the $5 \sigma$ detection limit (90 mJy) for a $1.5 \mathrm{~m}$ telescope at $600 \mathrm{GHz}$ (right).

This has driven extensive searches for high redshift galaxy proto-clusters in the past two decades (for a review see Overzier, 2016). Classical systematic cluster searches were carried out via X-ray (e.g., Mehrtens et al., 2012; Willis et al., 2013), Sunyaev-Zeldovich (SZ; Sunyaev and Zeldovich, 1972; Bleem et al., 2015; Planck Collaboration XXVII, 2016; Hilton et al., 2018) and optical/near-infrared (Rykoff et al., 2016; Wen and Han, 2018; Gonzalez et al., 2019; Maturi et al., 2019) surveys. These surveys, however, have yielded only a handful of confirmed proto-cluster detections at $z \gtrsim 1.5$ (Overzier, 2016). The reason is that, while at $z \lesssim 1-1.5$ cluster cores are dominated by passive early-type galaxies and are filled by hot gas, at higher $z$ cluster members enter the dust-obscured star-formation phase and the intergalactic gas is no longer necessarily at the virial temperature. In fact, several programs have found an inversion of the star formation-density relation at $z \gtrsim 1.3$. At lower redshifts, it has long been known that there is a well defined increase of the passive elliptical and lenticular (denoted S0) population with increasing density (Dressler, 1980). Dense cluster cores are preferentially populated by massive, passively evolving, early-type galaxies. Star-forming galaxies are generally found in the cluster outskirts and in the field ${ }^{4}$. At higher redshifts, however, cluster cores are found to have an increasing population of strongly star-forming, luminous infrared galaxies (Alberts et al., 2014, 2016; Wagner et al., 2017). Their specific SFR increases rapidly from $z \sim$ 0.2 to $z \sim 1.3$, mostly driven by the activation of star formation in early-type galaxies. The star-formation activity in cluster core $(r<0.5 \mathrm{Mpc})$ galaxies reaches the field level at $z \gtrsim 1.2$.

So far, most of the detections of high- $z$ (proto-)clusters have been obtained either as by-products of large spectroscopic or multi-band photometric surveys, or using biased tracer techniques (Overzier, 2016). The latter techniques consists in targeting the immediate environment of tracers of massive forming systems, like high redshift radio galaxies and QSOs, Ly $\alpha$ blobs and bright sub-millimeter galaxies. The data sets collected in these ways are obviously highly heterogeneous, affected by strong, hard to quantify selection biases and only sparsely sample the redshift distribution of (proto-)clusters. They are therefore

${ }^{4}$ Field galaxies are those that do not reside in overdense regions. 
unsuitable to obtain a complete picture of the build-up of galaxy clusters over cosmic time.

The very fact that member galaxies are increasingly infrared luminous with increasing redshift, means that (sub-)mm surveys are the most effective tool to detect high- $z$ proto-clusters. They are rare objects and therefore the multi-steradian $\mathrm{CMB}$ surveys are optimal to find at least the (sub-)mm brightest ones. A blind search on Planck maps was carried out by Planck Collaboration Int. XXXIX (2016) at 5' resolution. They looked for intensity peaks with "cold" sub-mm colors, i.e., with continuum spectra peaking between 353 and $857 \mathrm{GHz}$, consistent with redshifts $z>2$ for typical dust emission spectra. Herschel/SPIRE follow-up of 234 Planck targets with such colors showed that almost all of them correspond to strong over-densities of red 350 and $500 \mu \mathrm{m}$ sources in comparison to reference SPIRE fields (Planck Collaboration Int. XXVII, 2015). Further investigations of Planck proto-cluster candidates were carried out by Clements et al. (2014), Clements et al. (2016), and Greenslade et al. (2018).

However, the Planck's angular resolution of $\simeq 5^{\prime}$, corresponding to a physical size of about $2.5 \mathrm{Mpc}$ at $z=1.5-2$, is not optimal for detecting proto-clusters. By means of detailed simulations based on a physically motivated galaxy evolution model, Negrello et al. (2017b) showed that essentially all Planck's cold peaks can be interpreted as positive Poisson fluctuations of the number of high- $z$ proto-clusters of dusty galaxies within the Planck beam, rather than being individual clumps of physically bound galaxies.

The study (Alberts et al., 2014) of 274 clusters with $0.3 \leq$ $z \leq 1.5$ from the Spitzer InfraRed Array Camera (IRAC) Shallow Cluster Survey, using Herschel/SPIRE 250- $\mu$ m imaging, showed that the density of IR-emitting cluster members clearly exceeds that of the background field level only within $0.5 \mathrm{Mpc}$ of the cluster center. A linear scale of $0.5 \mathrm{Mpc}$ corresponds to an angular scale of about $1^{\prime}$ at redshifts in the range 1.5-2.5, close to the PICO/CORE/CMB Bharat FWHM at $800 \mathrm{GHz}$ and to the resolution of CMB-S4 and of the Simons Observatory at $\mathrm{mm}$ wavelengths. Thus, next generation $\mathrm{CMB}$ experiments will be optimally suited to detect the bright cluster cores of the kind discovered by Ivison et al. (2013) at $z=2.41$, Wang et al. (2016) at $z=2.51$, Miller et al. (2018) at $z=4.31$, and Oteo et al. (2018) at $z=4.0$ (see Figure 6). Spectroscopic confirmations of additional proto-clusters detected at sub- $\mathrm{mm}$ wavelengths have been most recently reported by Gómez-Guijarro et al. (2019) and Lacaille et al. (2018). Figure 6 shows that proto-clusters bright enough to be detected by next generation CMB experiments exist out to $z \gtrsim 4$. Note that, as argued by De Zotti et al. (2018), proto-clusters stand out as intensity peaks in backgroundsubtracted maps more clearly than in surveys of point sources. This is because such intensity peaks include the contribution of all member galaxies, including those below the point source detection limit, that may dominate the integrated flux density.

How many dusty proto-cluster detections can we expect? The predictions of the model by Negrello et al. (2017b), that reproduced all the relevant data, are shown in Figure 7. Spaceborne experiments like PICO/CORE/CMB Bharat will detect many tens of thousands of these objects, with a predicted redshift distribution peaking at $z \simeq 2$ and extending out to $z \simeq 4.5$. Ground based instruments will preferentially detect proto-clusters at higher redshifts, out to $z \gtrsim 5$, with a distribution having a broad peak around $z \simeq 2.75$. Since high- $z$ clusters are expected to be rare, the number of detections will be of only $\simeq 10^{-2} \mathrm{deg}^{-2}$ at the SPT completeness limit at $1.4 \mathrm{~mm}$. The number of detections rapidly increase with decreasing detection limit, reaching $\simeq 40 \mathrm{deg}^{-2}$ at the SPT confusion limit. This will constitute a real breakthrough in the observational determination of the formation history of the cluster-sized dark matter halos. Follow-up observations will characterize the properties of member galaxies, probing the galaxy evolution in dense environments and shedding light on the complex physical processes driving it.

$\mathrm{CMB}$ experiments will also allow us to investigate the evolution of galaxy populations in clusters detected by other means, e.g., via their X-ray emission or via the thermal SZ effect. De Zotti et al. (2018, their Figure 4) showed that space-borne $\mathrm{CMB}$ experiments with telescopes of the $1.5 \mathrm{~m}$ class will detect, at $800 \mathrm{GHz}$ the dust emission of the brightest $M \simeq 10^{14} \mathrm{M}_{\odot}$ clusters and of typical $M \simeq 10^{14.5} \mathrm{M}_{\odot}$ clusters at $1 \lesssim z \lesssim 1.5$. More massive clusters will be detected over broader redshift ranges. Figure 8 shows that SPT-like ground based surveys observing at $220 \mathrm{GHz}$ will perform similarly or only slightly worse. For comparison, Herschel has allowed the study of the IR emission from clusters up to $z \simeq 1.7$ (Alberts et al., 2016), but the sample comprises only 11 clusters. The Herschel data have shown large variations in cluster properties, highlighting the need for evolutionary studies of large, uniform cluster samples over a

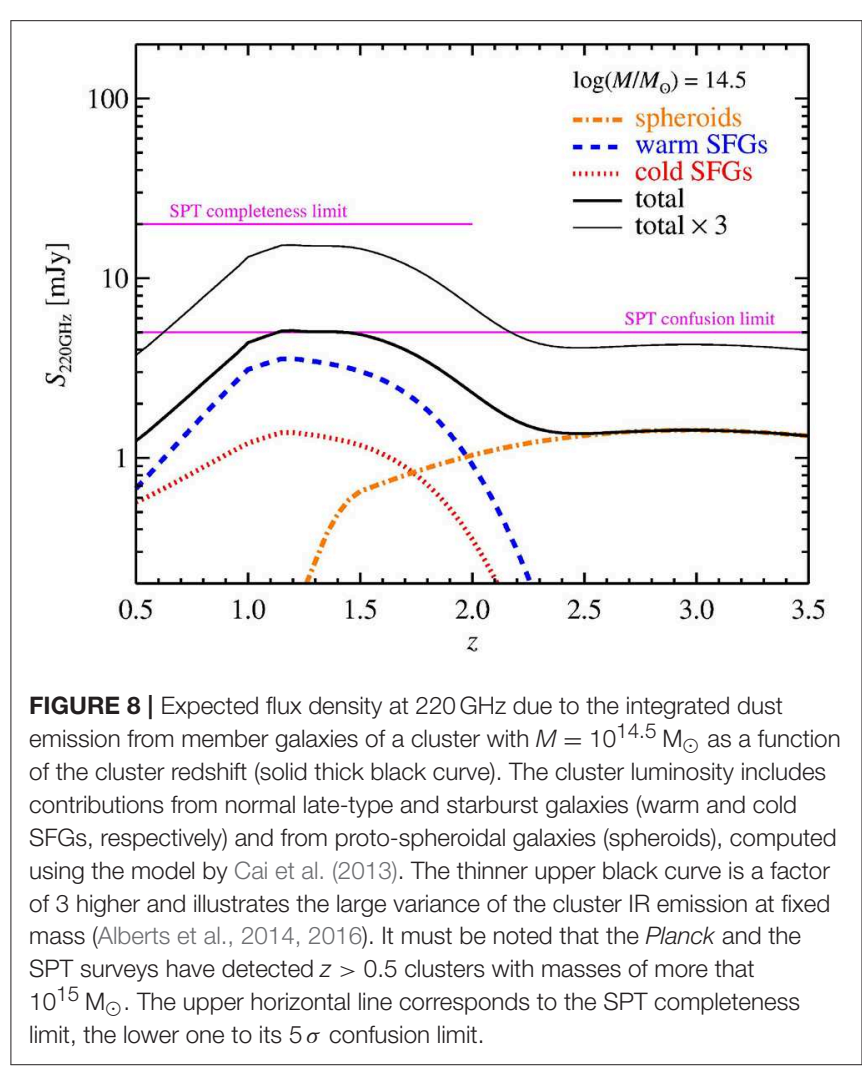


broad redshift range. Next generation CMB experiments will fulfill this need. Stacking will allow us to carry out a statistical investigation of the evolution of the cluster IR emission to fainter levels. Targets for stacking will abound. eROSITA (extended ROentgen Survey with an Imaging Telescope Array) will provide an all-sky deep X-ray survey detecting $\sim 10^{5}$ galaxy clusters out to $z>1$ (Merloni et al., 2012). CMB experiments themselves will detect tens of thousands of clusters via the SZ effect (Abazajian et al., 2016; Melin et al., 2018b; Ade et al., 2019; Hanany et al., 2019).

This will be important to investigate the evolution of the specific SFR in dense environments. In addition, the dust emission in galaxy clusters will impact the completeness of SZ surveys and will distort the SZ signal (Melin et al., 2018a), affecting the cosmological results derived from SZ observations. While the latter effect was shown to be negligible in Planck's case (Melin et al., 2018a), it will be important for the much deeper next generation surveys.

\section{RADIO SOURCES}

\subsection{Blazar Physics}

Although substantial progress on the characterization of $\mathrm{mm}$ and sub-mm properties of extragalactic radio sources has been made in recent years mainly thanks to surveys with WMAP, Planck, the SPT and the ACT, the available information is still limited. The overwhelming majority of extragalactic radio sources detectable in the frequency range of $\mathrm{CMB}$ experiments are blazars, i.e., sources whose radio emission is dominated by relativistic jets collimated by intense magnetic fields and closely aligned with the line of sight. These objects with extreme properties are of special interest since they are also strong gamma-ray sources: about $90 \%$ of the firmly identified extragalactic gamma-ray sources are blazars.

Accurate source counts over large flux density intervals provide key constraints on evolutionary models of these sources. Just because high frequency surveys are still far less extensive

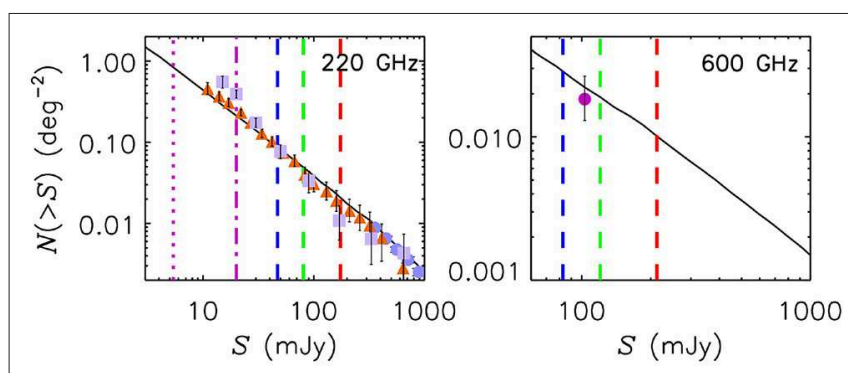

FIGURE 9 | Integral number counts of radio sources at 220 and $600 \mathrm{GHz}$. The vertical dashed lines show the $5 \sigma$ confusion limits for a space-borne experiment with a $1 \mathrm{~m}, 1.5 \mathrm{~m}$, and a $2 \mathrm{~m}$ telescope (from right to left) operating at the diffraction limit. The dot-dashed and dotted vertical lines on the left panel show the completeness limit of the SPT survey and the $5 \sigma$ confusion limit for the SPT telescope, respectively. The data points on the left panel are from Mocanu et al. (2013, SPT, orange triangles), Marsden et al. (2014, ACT, lavender squares), Planck Collaboration XIII (2011, light blue circles); the data point on the right panel is from Bonato et al. (2019b) and is based on Herschel survey data. The solid black lines are predictions of the Tucci et al. (2011) model. than those at low radio frequencies, evolutionary models for blazar populations, Flat Spectrum Radio Quasars (FSRQs) and BL Lacertae sources (BL Lacs), are far less advanced than those for steep-spectrum radio sources. For example, while clear evidence for downsizing ${ }^{5}$ was reported in the case of steep-spectrum sources (Massardi et al., 2010; Rigby et al., 2015), the available data are insufficient to test if this is the case also for FSRQs; for BL Lacs the constraints on evolutionary parameters are even weaker. This situation hampers sharp tests of unified models.

Planck has already provided strong indications of the crucial role of blazar photometry up to sub-mm wavelengths to get information on the energy spectrum of relativistic electrons responsible for the synchrotron emission, with interesting implications for the acceleration mechanisms (Planck Collaboration XLV, 2016).

Another interesting open question is the geometry of the emitting regions. The most commonly used model for the spectral energy distribution (SED) of compact, radio loud Active Galactic Nuclei (AGNs) is a leptonic, one-zone model, where the emission originates in a single component. The SEDs typically consist of two broad-band bumps; the one at lower frequencies is attributed to synchrotron radiation while the second, peaking at gamma-ray energies, is attributed to inverse Compton. The one-zone model is generally found to provide an adequate approximation primarily because of the limited observational characterization of the synchrotron SED, with fragmentary data over a limited frequency range. However VLBI images show multiple knots often called "components" of the jet. The standard model (Marscher and Gear, 1985) interprets the knots as due to shocks that enhance the local synchrotron emission.

The spectrum is explained as the result of the superposition of different synchrotron self-absorbed components in a conical geometry. The synchrotron self-absorption optical depth scales as $\tau_{\text {sync }} \propto B_{\perp}^{(p+2) / 2} v^{-(p+4) / 2}$ where $B_{\perp}$ is the magnetic field component perpendicular to the electron velocity and $p$ is spectral index of the energy distribution of relativistic electrons (typically, $p \simeq 2.5$ ). Thus $\tau_{\text {sync }}$ increases toward the nucleus as the magnetic field intensity and its ordering increases, but is strongly frequency dependent: the emission at higher and higher frequencies comes from smaller and smaller distances from the central engine.

Thus the $\mathrm{mm}$ and sub-mm emissions provide information on the innermost regions of the jets, where it is optically thin, while the emission at longer wavelengths is affected by selfabsorption. Interestingly millimeter-wave flux densities of FSRQs turn out to be strongly correlated with simultaneous gammaray fluxes (Fuhrmann et al., 2016; Fan and $\mathrm{Wu}, 2018$ ). The strongest gamma-ray flares were found to occur during the rising/peaking stages of millimeter flares. This suggests that the gamma-ray flares originate in the millimeter-wave emitting regions of these sources.

The available data are mostly at $\mathrm{cm}$ (or longer) wavelengths and are scanty at (sub-)mm wavelengths because of the limited sky areas covered by the available surveys. Ground-based

\footnotetext{
5 "Downsizing" refers to the very different evolutionary behavior of high- and lowluminosity sources, in the sense that the redshift of the peak space density of sources decreases with luminosity.
} 
experiments with $\sim 6 \mathrm{~m}$ telescopes, like CMB-S4 and the Simons Observatory, will detect thousands of blazars per sr at millimeter wavelengths (Figure 9). Extrapolating the $8.4 \mathrm{GHz}$ flux densities of the $18 z>4$ FSRQs listed by Caccianiga et al. (2019) using the measured $1.4-8.4 \mathrm{GHz}$ spectral indices we find that a large fraction of them will be detected by these experiments, including the highest redshift blazar known, GB6J090631 + 693027 at $z=5.47$. The radio selection, being unaffected by obscuration, provides an unbiased census at least of the radio-loud fraction of high- $z$ AGNs.

The most luminous high- $z$ FSRQs were found to have black holes with the largest masses, up to $\simeq 4 \times 10^{10} M_{\odot}($ S5 $0014+$ 813 at $z=3.366$; Ghisellini et al., 2013). Such objects have particularly hard mm-wave spectra, are rare and bright because of the Doppler boosting of their flux densities. CMB surveys are thus optimally suited to detect them. Since the flux boosting occurs for jets closely aligned with the line of sight $(\theta<1 / \Gamma$, $\Gamma \sim 15$ being the bulk Lorentz factor), for each FSRQ there are other $2 \Gamma^{2}$ (i.e., hundreds) sources of similar intrinsic properties but pointing elsewhere. This means that blazars are very efficient probes of extreme super-massive black holes at high $z$.

Very large black hole masses at high $z$ are puzzling because it is challenging to grow a stellar mass seed black hole to $>10^{9} M_{\odot}$ in the limited age of the universe. It is even more challenging in the case of jetted quasars because it is commonly believed that the jets are associated with rapidly spinning black holes. But then the radiative efficiency is large and the mass growth is slower. Yet at least 4 FSRQs has been discovered at $z>5$; one of them (SDSS J013127.34 - 032100.1 at $z=5.18$ ) has estimated black hole mass of $\simeq 1 \times 10^{10} M_{\odot}$ (Ghisellini et al., 2015).

Next-generation space-borne CMB experiments with $\simeq 1.5 \mathrm{~m}$ telescopes, like PICO, will provide, for the first time, samples of hundreds of blazars blindly selected at sub-mm wavelengths. An important property of surveys from space is that they provide simultaneous photometry over a broad frequency range, thus overcoming the complications due to variability and allowing us to directly connect the observed SED to the physical processes operating along the jet.

\subsection{Earliest and Latest Phases of Radio Activity}

Large-area surveys at frequencies of tens to hundreds $\mathrm{GHz}$ will also detect the rare but very interesting sources associated to the earliest and to the latest stages of the radio-AGN evolution, both characterized by emissions peaking in this frequency range (De Zotti et al., 2005). It is now widely agreed that extreme gigahertz peaked spectrum (GPS) sources correspond to the early stages of the evolution of powerful radio sources, when the radio emitting region grows and expands within the interstellar medium of the host galaxy, before plunging in the inter-galactic medium and becoming an extended radio source. There is a clear anti-correlation between the peak (turnover) frequency and the projected linear size of GPS sources, suggesting a decrease of the peak frequency as the emitting blob expands. The identification of these sources is therefore a key element in the study of the early evolution of radio AGNs. High-frequency surveys will detect these sources very close to the moment when they turn on.

Possible examples of extremely young sources are the six narrow-line Seyfert 1 galaxies detected by Lähteenmäki et al. (2018) at $37 \mathrm{GHz}$ with flux densities of 270-970 mJy but undetected by the FIRST survey, complete down to $\simeq 1 \mathrm{mJy}$ at $1.4 \mathrm{GHz}$, carried out about $20 \mathrm{yr}$ ago. One possibility is that the new observations have discovered newly triggered radio activity from nuclei that were essentially radio silent two decades ago.

Young radio activity was recently discovered by Bruni et al. (2019) in the nuclei of 8 out of $13(\simeq 61 \%)$ hard X-ray selected giant radio galaxies for which they had enough spectral coverage to ascertain the presence of a peak. Two of the 8 sources have a peak frequency $>10 \mathrm{GHz}$ and at least one is bright enough to be clearly detected by next generation CMB experiments.

The multi-frequency surveys by next generation CMB experiments will provide an unbiased view of the frequency of these phenomena and will measure their high frequency SEDs, shedding light on their nature. These data will enable studies of the launching of relativistic jets as well as of the evolutionary paths that young AGNs take on their way to becoming fullyevolved, powerful radio sources.

Large area (sub-)mm surveys will also allow us to investigate the late stages of the AGN evolution in elliptical galaxies, characterized by low radiation/accretion efficiency. These manifest themselves via a nuclear radio emission described by advection-dominated accretion flows (ADAFs) and/or by adiabatic inflow-outflow solutions (ADIOS). Doi et al. (2005) have found that at least half of their sample of 20 low-luminosity AGNs with compact radio cores show radio spectra rising at least up to $96 \mathrm{GHz}$, consistent with the "sub-millimetre bump" predicted by an ADAF model. Again CMB surveys will determine the abundance of these objects and their SED measurements will clarify the origin of the emission.

Predictions of the expected number of detections of early and late phases of radio activity are limited to $\leq 30 \mathrm{GHz}$ (De Zotti et al., 2005; Tinti and De Zotti, 2006). They suggest that at 20-30 $\mathrm{GHz}$ hundreds of these objects can be detected by ground-based experiments. Space-borne experiments are confusion-limited to much brighter flux densities, implying a detection rate at least one order of magnitude lower. The number of detections is predicted to drop rapidly with increasing frequency (Blandford and McKee, 1976; Granot and Sari, 2002).

\subsection{The Extragalactic Transient Sky}

High-sensitivity and high-angular-resolution CMB surveys also offer a unique opportunity to carry out an unbiased investigation of the largely unexplored extragalactic $\mathrm{mm} / \mathrm{sub}-\mathrm{mm}$ transient sky (Metzger et al., 2015). So far measurements have been limited to follow-up of objects detected at other wavelengths, with limited success partly because of the need for excellent weather conditions or because the events were too short-lived to detect without very rapid reaction times. $\mathrm{CMB}$ surveys will allow us to discover new, unknown transient sources in this band.

One example of transient phenomena are outbursts from AGNs and especially from blazars. Outbursts and, more generally, variability, provide key information on the flow of the 
plasma within the relativistic jets. Signatures of evolving shocks in the strongest radio flares were seen by Planck Collaboration XLV (2016) although high frequency light curves are generally quite similar (approximately achromatic variability). These results are compatible with the standard shocked jet model, but other interpretations are possible. Definite conclusions are currently hampered by the limited statistics. This limit will be overcome by next generation CMB experiments from space which will provide multi-epoch simultaneous observations of large blazar samples over a broad frequency range. This will allow us to study their variability properties as a function of flux density and spectral shape.

Perhaps even more interesting is the possibility of detecting radio aftergrows of gamma-ray bursts (GRBs). Afterglows often have a spectral peak in or near the $\mathrm{mm}$ range (Granot and Sari, 2002), with emission lasting over days to weeks. One candidate object with properties broadly consistent with a GRB afterglow was tentatively detected by Whitehorn et al. (2016) on SPT data over $100 \mathrm{deg}^{2}$ with an observing time of $6,000 \mathrm{~h}$, but the statistical significance of the detection was too low to completely rule out a fluctuation. GRB emission is expected to be less tightly beamed at these wavelengths than in gamma-rays. Thus afterglows not accompanied by detectable gamma-ray emission are expected to exist, but have not been detected yet. Blind surveys of large sky areas by next generation ground-based CMB experiments down to $\simeq 10 \mathrm{mJy}$ sensitivity can reveal these orphan afterglows and new, unknown sources. Multiple detections per year are expected. Even a non-detection will place interesting constraints on the shock dynamics and on the energy budget of the unknown GRB progenitors.

One example of unexpected phenomena that may show up at (sub-)mm wavelengths is the extraordinary extragalactic transient AT2018cow, with an estimated peak flux density of 94 mJy at $\simeq 100 \mathrm{GHz}$ (Ho et al., 2019). This object may herald a new class of energetic transients which at early times are most readily observed at (sub-)mm wavelengths.

\section{DETECTING SOURCES IN POLARIZATION}

Accurate simulations (Remazeilles et al., 2018) showed that, for a tensor-to-scalar ratio $r \simeq 10^{-3}$ (i.e., at levels predicted by models currently of special interest, such as Starobinsky's $R^{2}$ and Higgs inflation), the overall uncertainty on $r$ is dominated by foreground residuals and that unresolved polarized point sources can be the dominant foreground contamination over a broad range of angular scales $(\ell \gtrsim 50)$. A thorough understanding of the polarization properties of extragalactic sources is therefore crucial.

While the point source power spectra in total intensity are quite well constrained, estimates in polarization are obtained by coupling the counts in total intensity with distributions of the polarization fractions derived from lower frequency surveys. This implicitly assumes that such distributions are frequencyindependent. Although this assumption is consistent with the available data (Battye et al., 2011; Galluzzi et al., 2018), there are substantial uncertainties and indeed variations are expected since emissions at different frequencies of flat-spectrum sources, that dominate in the relevant frequency range, come from different regions where the magnetic field properties are expected to be different.

Polarization measurements at $\gtrsim 100 \mathrm{GHz}$ are still scanty. Only a few tens of sources (all radio) were detected by Planck in the "extragalactic zone" (De Zotti et al., 2018). Galluzzi et al. (2019) carried out ALMA high sensitivity polarimetric observations at $97.5 \mathrm{GHz}$ of a complete sample of 32 compact extragalactic radio sources brighter than $200 \mathrm{mJy}$ at $20 \mathrm{GHz}$; a detection rate of $97 \%$ was achieved.

Datta et al. (2019) reported the detection of linear polarization at $148 \mathrm{GHz}$ of 26 extragalactic sources with total flux density $S_{148 \mathrm{GHz}}>215 \mathrm{mJy}, 14$ of which at greater than $3 \sigma$ significance, during the first two seasons of the ACT polarization (ACTPol) survey, covering $680 \mathrm{deg}^{2}$ of the sky. Their results are consistent with a mean fractional polarization $\langle\Pi\rangle=0.028 \pm 0.005$ and a standard deviation $\sigma_{\mathrm{p}}=0.054$, independent of total intensity.

Gupta et al. (2019) investigated the polarization properties of extragalactic sources in the SPTpol $500 \mathrm{deg}^{2}$ survey at 95 and $150 \mathrm{GHz}$ down to $6 \mathrm{mJy}$. They found no evidence that the polarization fraction depends on flux density. Assuming $\langle\Pi\rangle$ to be constant across flux bins they obtained, for radio sources, flux weighted $\left\langle\Pi^{2}\right\rangle=(8.9 \pm 1.1) \times 10^{-4}$ at $95 \mathrm{GHz}$ and $\left\langle\Pi^{2}\right\rangle=$ $(6.9 \pm 1.1) \times 10^{-4}$ at $150 \mathrm{GHz}$.

These results are consistent with the conclusion by Bonavera et al. (2017a) who applied stacking techniques to radio sources detected by Planck in total intensity, finding an average fractional polarization of $\simeq 3 \%$, essentially independent of frequency from 30 to $353 \mathrm{GHz}$. A similar conclusion was reached by the independent analysis of Trombetti et al. (2018).

The (sub-)mm polarization properties of dusty galaxies are essentially unknown. The only published measurement of the polarization degree averaged over the whole galaxy (Greaves and Holland, 2002) yielded $\Pi \simeq 0.4 \%$ at $850 \mu \mathrm{m}$ for the prototype starburst galaxy M 82. A 99\% confidence upper limit of $1.54 \%$ at the same wavelength was reported by Seiffert et al. (2007) for the ultraluminous infrared galaxy (ULIRG) Arp 220. From the Planck dust polarization maps of the Milky Way, De Zotti et al. (2018) found an average value of the Stokes Q parameter of about $2.7 \%$. If this is typical of disk galaxies, their mean polarization degree averaged over all possible inclination angles is $\simeq 1.4 \%$.

By applying the stacking techniques to a large sample of dusty galaxies drawn from the PCCS2 $857 \mathrm{GHz}$ catalog, Bonavera et al. (2017b) estimated a median $\Pi$ of $(2.0 \pm 0.8 \%)$ at 353 $\mathrm{GHz}$, consistent with the $90 \%$ confidence upper limit of $2.2 \%$ derived by Trombetti et al. (2018). The sample by Gupta et al. (2019) includes 55 sources classified as dusty galaxies. No polarization signal was detected for these sources. The resulting 95\% confidence level upper limits are $\left\langle\Pi^{2}\right\rangle<16.9 \times 10^{-3}$ at $95 \mathrm{GHz}$ and $\left\langle\Pi^{2}\right\rangle<2.6 \times 10^{-3}$ at $150 \mathrm{GHz}$, consistent with earlier results.

These low values of $\Pi$ are understood as due to the complex structure of galactic magnetic fields, with reversals along the line of sight, and to the disordered alignment of dust grains; both effects work to decrease the global polarization 
degree. Nevertheless, the current limits on the distribution of polarization fractions of dusty galaxies permit a contamination of $\mathrm{CMB}$ polarization maps comparable to that of radio sources down to $100-140 \mathrm{GHz}$, and dominant at higher frequencies (Bonavera et al., 2017b; Trombetti et al., 2018). The amplitude of the power spectrum of polarized dusty galaxies may be close to the level of $C M B$ lensing $B$-modes and of primordial $B$-modes for $r \simeq 0.01$.

At variance with total intensity, in the case of polarization the detection limit is dictated by sensitivity, not by confusion noise. Hence, the spectacular improvement in sensitivity of next generation $\mathrm{CMB}$ experiments, compared to Planck, will allow a real breakthrough in the characterization of the polarization properties of extragalactic sources. Figure 10 shows the integral number counts in polarized intensity of radio sources and of dusty galaxies at 220 and $800 \mathrm{GHz}$ obtained from the simulations described in De Zotti et al. (2018). The vertical dashed black lines show the $5 \sigma$ detection limits for a space-borne instrument with a $1.5 \mathrm{~m}$ telescope and state-of-the-art sensitivity, derived from simulations made as described in De Zotti et al. (2018), assuming log-normal distributions of the polarization fractions. For radio sources we adopted the same mean and dispersion $(2.14 \%$ and of $0.9 \%$, respectively) used by De Zotti et al. (2018). For dusty galaxies we adopted the value for our own Galaxy, averaged over inclinations (1.4\%), with a dispersion of $1 \%$. The estimated $5 \sigma$ detection limits are $3.7 \mathrm{mJy}$ at $220 \mathrm{GHz}$ and $6.2 \mathrm{mJy}$ at $800 \mathrm{GHz}$. At these limits the expected integral counts at $220 \mathrm{GHz}$ are of $\simeq 160 \mathrm{sr}^{-1}$ radio sources and of $\simeq 100 \mathrm{sr}^{-1}$ dusty galaxies; at $800 \mathrm{GHz}$ they are of $\simeq 50 \mathrm{sr}^{-1}$ radio sources and of $\simeq$ $36,000 \mathrm{sr}^{-1}$ dusty galaxies.

Under these assumptions, radio sources are the dominant population below $\simeq 200 \mathrm{GHz}$. A comparison with the results reported by De Zotti et al. (2018) who used a mean polarization fraction of $0.5 \%$ for dusty galaxies shows that the predicted

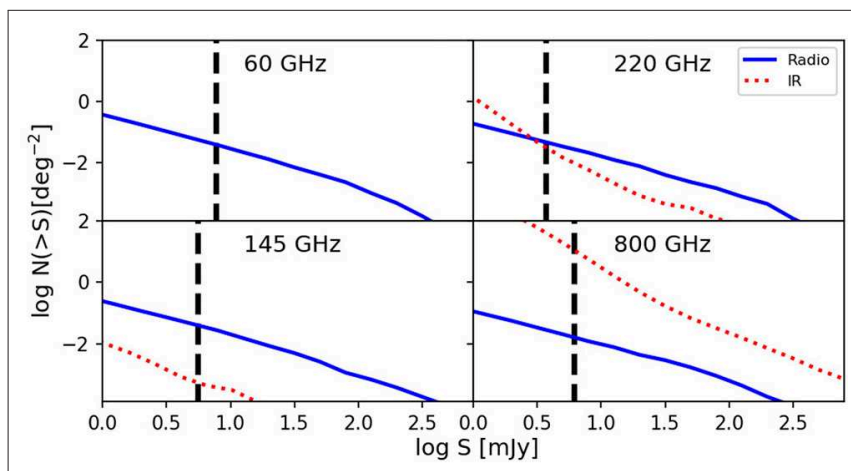

FIGURE 10 | Estimated integral number counts in polarized intensity of radio sources and of dusty galaxies (IR) at $60,145,220$, and $800 \mathrm{GHz}$. The contribution of dusty galaxies is completely negligible at $60 \mathrm{GHz}$. The vertical dashed black lines show the $5 \sigma$ detection limits for a space-borne instrument with a $1.5 \mathrm{~m}$ telescope and state-of-the-art sensitivity derived from simulations similar to those described in De Zotti et al. (2018). The present simulations assumed log-normal distributions of the polarization fractions with mean and dispersion of $2.14 \%$ and of $0.9 \%$, respectively, for radio sources and of 1.4 and $1 \%$, respectively, for dusty galaxies. counts are highly sensitive to the choice for this, highly uncertain, quantity.

As illustrated by Figure 10, next generation CMB experiments will be capable of providing, for the first time, direct counts in polarization both for radio sources and for dusty galaxies, thus overcoming the current large uncertainties on the source power spectra in polarization. On one side this will allow a much better control of the extragalactic source contamination of CMB maps. This is particularly important in the $60-120 \mathrm{GHz}$ frequency range, where diffuse polarized foreground emissions display a broad minimum.

On the other side, polarization observations enable us to understand geometrical structure and intensity of magnetic fields, particle densities and structures of emission regions. In the case of extragalactic radio sources, emission at $\mathrm{mm} / \mathrm{sub}-\mathrm{mm}$ wavelengths is synchrotron radiation arising close to the origin of the jet, on sub-parsec scales, generally unresolved even by the highest frequency very long baseline interferometry (VLBI) maps (Nartallo et al., 1998). At these wavelengths the emission is expected to be optically thin, so that self-absorption and Faraday rotation are negligible. Then, in principle, the linear polarization degree can be as high as $60-80 \%$ if the magnetic field is ordered (Saikia and Salter, 1988). Hence, measurements of the polarization degree constrain the magnetic field geometry.

\section{CONCLUSIONS}

Thanks to their high sensitivity and to the coverage of large fractions of the sky, next generation $\mathrm{CMB}$ experiments will provide ground-breaking results in the field of extragalactic astrophysics. They will provide samples of several thousands of the brightest high- $z$ strongly lensed, dusty galaxies, with extreme amplifications, up to $\mu \simeq 50$, and out to $z \simeq 6$. This will constitute an ideal data-base for high-resolution follow-up observations addressing the internal structure and kinematics of primordial galaxies, i.e., to understand the physical processes that drive the galaxy formation and early evolution across a broad redshift range, up to the re-ionization epoch.

CMB experiments will also provide unbiased, flux limited samples of tens of thousands of dense proto-cluster cores out to $z \gtrsim 4$, well beyond the reach of classical (optical, X-ray, SZ) cluster surveys that are mostly limited to $z \lesssim 1.5$. They will effectively open a new window on the study of early phases of cluster formation, when their member galaxies were actively star forming and before the hot intergalactic medium was in place. This is crucial to observationally assess the formation history of the most massive dark matter halos, traced by clusters, a critical test of models for structure formation.

These experiments will also allow us to investigate, via direct detections complemented with stacking analyses, the evolution of the star-formation rate in virialized galaxy clusters detected by X-ray and SZ surveys (including those carried out by the experiments themselves), shedding light on the role of dense environments on galaxy evolution.

The data on radio sources will greatly improve our understanding of the evolutionary properties of FSRQs and BL 
Lac objects. They will also probe the jet physics in its innermost regions as well as the earliest and latest phases of radio activity. Particularly interesting is the possibility of getting an unbiased view of the abundance of candidate newly-born radio sources. These surveys also offer a unique opportunity to carry out an unbiased investigation of the largely unexplored $\mathrm{mm} / \mathrm{sub}$ $\mathrm{mm}$ transient sky, including the detection of predicted, but still unseen, "orphan" radio afterglows of GRBs as well as unexpected transient phenomena.

Furthermore, these experiments will provide the first, extensive, blind high-frequency census of the polarization properties of radio sources and of star-forming galaxies. This is essential to clean $\mathrm{CMB}$ maps at the level required to measure the faint primordial $B$-mode power spectrum. At the same time, $\mathrm{mm} / \mathrm{sub}-\mathrm{mm}$ polarization data on radio sources provide unique information on the geometry of the magnetic field on sub-pc scales, unresolved even by high-frequency VLBI observations. Polarimetry of dusty galaxies is informative on the structure of galactic magnetic fields.

\section{AUTHOR CONTRIBUTIONS}

GD has coordinated the work and written most of the text. $\mathrm{MB}, \mathrm{MN}$, and Z-YC have made model calculations and prepared Figures 2, 3, 5-9. TT and CB worked on the selection of strongly lensed galaxies detected by Planck and made Figure 4. DH and ML-C made the simulations and the source extraction on degraded SPT maps and produced Figure 1. Z-YC computed the redshift distribution of strongly lensed galaxies at $1.4 \mathrm{~mm}$ and

\section{REFERENCES}

Abazajian, K. N., Adshead, P., Ahmed, Z., Allen, S. W., Alonso, D., Arnold, K. S., et al. (2016). CMB-S4 Science Book, First Edition.

Ade, P., Aguirre, J., Ahmed, Z., Aiola, S., Ali, A., Alonso, D., et al. (2019). The simons observatory: science goals and forecasts. J. Cosmol. Astropart. Phys. 2:056. doi: 10.1088/1475-7516/2019/02/056

Alberts, S., Pope, A., Brodwin, M., Atlee, D. W., Lin, Y.-T., Dey, A., et al. (2014). The evolution of dust-obscured star formation activity in galaxy clusters relative to the field over the last 9 billion years. Month. Notices RAS 437, 437-457. doi: 10.1093/mnras/stt1897

Alberts, S., Pope, A., Brodwin, M., Chung, S. M., Cybulski, R., Dey, A., et al. (2016). Star formation and AGN activity in galaxy clusters from $\mathrm{z}=1$ 2: a multi-wavelength analysis featuring herschel/PACS. Astrophys. J. 825:72. doi: 10.3847/0004-637X/825/1/72

Aravena, M., Murphy, E. J., Aguirre, J. E., Ashby, M. L. N., Benson, B. A., Bothwell, M., et al. (2013). Large gas reservoirs and free-free emission in two lensed star-forming galaxies at $\mathrm{z}=2.7$. Month. Notices RAS 433, 498-505. doi: 10.1093/mnras/stt741

Battye, R. A., Browne, I. W. A., Peel, M. W., Jackson, N. J., and Dickinson, C. (2011). Statistical properties of polarized radio sources at high frequency and their impact on cosmic microwave background polarization measurements. Month. Notices RAS 413, 132-148. doi: 10.1111/j.1365-2966.2010.1 8115.x

Blain, A. W., and Longair, M. S. (1993). Submillimetre cosmology. Month. Notices RAS 264, 509-521. doi: 10.1093/mnras/264.2.509

Blandford, R. D., and McKee, C. F. (1976). Fluid dynamics of relativistic blast waves. Phys. Fluids 19, 1130-1138. doi: 10.1063/1.861619 the integrated flux density of cluster galaxies at $220 \mathrm{GHz}$ as a function of redshift, and made a panel of Figures 3, 8. LB and JG-N performed the simulations used to determine the detection limits in polarization and made Figure 10. All authors critically reviewed the entire paper.

\section{FUNDING}

GD, CB, and TT acknowledge financial support from ASI/INAF agreement n. 2014-024-R.1 for the Planck LFI Activity of Phase E2 and from the ASI/Physics Department of the university of Roma-Tor Vergata agreement n. 2016-24-H.0 for study activities of the Italian cosmology community. MB acknowledges support from the Italian Ministero dell'Istruzione, Università e Ricerca through the grant Progetti Premiali 2012-iALMA (CUP C52I13000140001) and, together with CB and TT, from INAF under PRIN SKA/CTA FORECaST. DH acknowledges partial financial support from the Spanish Ministerio de Economía y Competitividad (MINECO) project AYA2015- 64508-P and from the RADIOFOREGROUNDS project, funded by the European Comission's H2020 Research Infrastructures under the Grant Agreement 687312.

\section{ACKNOWLEDGMENTS}

We are grateful to the referees for a careful reading of the manuscript and many useful comments. GD acknowledges enlightening discussions on CMB experiments with S. Hanany and J. Delabrouille.

Bleem, L. E., Stalder, B., de Haan, T., Aird, K. A., Allen, S. W., Applegate, D. E., et al. (2015). Galaxy clusters discovered via the Sunyaev-Zel'dovich effect in the 2500-square-degree SPT-SZ survey. Astrophys. J. Suppl. 216:27. doi: 10.1088/0067-0049/216/2/27

Bocquet, S., Dietrich, J. P., Schrabback, T., Bleem, L. E., Klein, M., Allen, S. W., et al. (2018). Cluster cosmology constraints from the $2500 \mathrm{deg}^{2}$ SPT-SZ survey: inclusion of weak gravitational lensing data from Magellan and the Hubble space telescope. arXiv[Preprint].arXiv:1812.01679.

Bonato, M., De Zotti, G., Leisawitz, D., Negrello, M., Massardi, M., Baronchelli, I., et al. (2019a). Origins space telescope: predictions for far-IR spectroscopic surveys. Publ. Astron. Soc. Aust. 36:e017. doi: 10.1017/pasa.2019.8

Bonato, M., Liuzzo, E., Herranz, D., González-Nuevo, J., Bonavera, L., Tucci, M., et al. (2019b). ALMA photometry of extragalactic radio sources. Month. Notices RAS 485, 1188-1195. doi: 10.1093/mnras/stz465

Bonavera, L., González-Nuevo, J., Argüeso, F., and Toffolatti, L. (2017a). Statistics of the fractional polarization of compact radio sources in Planck maps. Month. Notices RAS 469, 2401-2411. doi: 10.1093/mnras/stx1020

Bonavera, L., González-Nuevo, J., De Marco, B., Argüeso, F., and Toffolatti, L. (2017b). Statistics of the fractional polarization of extragalactic dusty sources in Planck HFI maps. Month. Notices RAS 472, 628-635. doi: $10.1093 / \mathrm{mnras} / \mathrm{stx} 2102$

Bruni, G., Panessa, F., Bassani, L., Chiaraluce, E., Kraus, A., Dallacasa, D., et al. (2019). A discovery of young radio sources in the cores of giant radio galaxies selected at hard X-rays. Astrophys. J. 875:88. doi: 10.3847/1538-4357/ ab1006

Caccianiga, A., Moretti, A., Belladitta, S., Della Ceca, R., Antón, S., Ballo, L., et al. (2019). The space density of $z>4$ blazars. Month. Notices RAS 484, 204-217. doi: $10.1093 / \mathrm{mnras} / \mathrm{sty} 3526$ 
Cai, Z.-Y., Lapi, A., and Xia, J.-Q. (2013). A hybrid model for the evolution of galaxies and active galactic nuclei in the infrared. Astrophys. J. 768:21. doi: 10.1088/0004-637X/768/1/21

Cañameras, R., Nesvadba, N., and Kneissl, R. e. a. (2017a). Planck's dusty GEMS. IV. Star formation and feedback in a maximum starburst at $z=3$ seen at 60 -pc resolution. Astron. Astrophys. 604:A117. doi: 10.1051/0004-6361/201630186

Cañameras, R., Nesvadba, N. P. H., and Guery, D., e. a. (2015). Planck's dusty GEMS: the brightest gravitationally lensed galaxies discovered with the Planck all-sky survey. Astron. Astrophys. 581:A105. doi: 10.1051/0004-6361/201425128

Cañameras, R., Nesvadba, N. P. H., and Kneissl, R. e. a. (2017b). Planck's dusty GEMS. III. A massive lensing galaxy with a bottom-heavy stellar initial mass function at $z=1.5$. Astron. Astrophys. 600:L3. doi: 10.1051/0004-6361/201630359

Cañameras, R., Nesvadba, N. P. H., Limousin, M., Dole, H., Kneissl, R., Koenig, S., et al. (2018). Planck's dusty GEMS. V. Molecular wind and clump stability in a strongly lensed star-forming galaxy at $z=2.2$. Astron. Astrophys. 620:A60. doi: 10.1051/0004-6361/201833679

Chown, R., Omori, Y., Aylor, K., Benson, B. A., Bleem, L. E., Carlstrom, J. E., et al. (2018). Maps of the southern millimeter-wave sky from combined $2500 \mathrm{deg}^{2}$ SPT-SZ and planck temperature data. Astrophys. J. Suppl. 239:10. doi: 10.3847/1538-4365/aae694

Cicone, C., Maiolino, R., Gallerani, S., Neri, R., Ferrara, A., Sturm, E., et al. (2015). Very extended cold gas, star formation and outflows in the halo of a bright quasar at $z>6$. Astron. Astrophys. 574:A14. doi: 10.1051/0004-6361/201424980

Clements, D. L., Braglia, F., Petitpas, G., Greenslade, J., Cooray, A., Valiante, E., et al. (2016). H-ATLAS: a candidate high redshift cluster/protocluster of star-forming galaxies. Month. Notices RAS 461, 1719-1733. doi: 10.1093/mnras/stw1224

Clements, D. L., Braglia, F. G., and Hyde, A. K. (2014). Herschel Multitiered Extragalactic Survey: clusters of dusty galaxies uncovered by Herschel and Planck. Month. Notices RAS 439, 1193-1211. doi: 10.1093/mnras/stt2253

Combes, F., Rex, M., Rawle, T. D., Egami, E., Boone, F., Smail, I., et al. (2012). A bright $z=5.2$ lensed submillimeter galaxy in the field of Abell 773. HLSJ091828.6+514223. Astron. Astrophys. 538:L4. doi: 10.1051/0004-6361/201118750

Cresci, G., and Maiolino, R. (2018). Observing positive and negative AGN feedback. Nat. Astron. 2, 179-180. doi: 10.1038/s41550-018-0404-5

Crocker, R. M., Krumholz, M. R., Thompson, T. A., and Clutterbuck, J. (2018). The maximum flux of star-forming galaxies. Month. Notices RAS 478, 81-94. doi: 10.1093/mnras/sty989

Datta, R., Aiola, S., Choi, S. K., Devlin, M., Dunkley, J., Dünner, R., et al. (2019). The atacama cosmology telescope: two-season ACTPol extragalactic point sources and their polarization properties. Month. Notices RAS 486, 5239-5262. doi: $10.1093 / \mathrm{mnras} / \mathrm{sty} 2934$

De Zotti, G., Castex, G., González-Nuevo, J., Lopez-Caniego, M., Negrello, M., Cai, Z.-Y., et al. (2015). Extragalactic sources in Cosmic Microwave Background maps. J. Cosmol. Astropart. Phys. 6:018. doi: 10.1088/1475-7516/20 15/06/018

De Zotti, G., González-Nuevo, J., Lopez-Caniego, M., Negrello, M., Greenslade, J., Hernández-Monteagudo, C., et al. (2018). Exploring cosmic origins with CORE: extragalactic sources in cosmic microwave background maps. J. Cosmol. Astropart. Phys. 4:020. doi: 10.1088/1475-7516/2018/04/020

De Zotti, G., Ricci, R., Mesa, D., Silva, L., Mazzotta, P., Toffolatti, L., et al. (2005). Predictions for high-frequency radio surveys of extragalactic sources. Astron. Astrophys. 431, 893-903. doi: 10.1051/0004-6361:20042108

Delabrouille, J., Betoule, M., Melin, J.-B., Miville-Deschênes, M.-A., GonzalezNuevo, J., Le Jeune, M., et al. (2013). The pre-launch Planck Sky Model: a model of sky emission at submillimetre to centimetre wavelengths. Astron. Astrophys. 553:A96. doi: 10.1051/0004-6361/201220019

Delabrouille, J., de Bernardis, P., Bouchet, F. R., Achúcarro, A., Ade, P. A. R., Allison, R., et al. (2018). Exploring cosmic origins with CORE: survey requirements and mission design. J. Cosmol. Astropart. Phys. 4:014.

Doi, A., Kameno, S., Kohno, K., Nakanishi, K., and Inoue, M. (2005). A highfrequency radio survey of low-luminosity active galactic nuclei. Month. Notices RAS 363, 692-704. doi: 10.1111/j.1365-2966.2005.09471.x

Dressler, A. (1980). Galaxy morphology in rich clusters - Implications for the formation and evolution of galaxies. Astrophys. J. 236, 351-365. doi: $10.1086 / 157753$
Eales, S., Dunne, L., Clements, D., Cooray, A., De Zotti, G., Dye, S., et al. (2010). The herschel ATLAS. Publ. ASP 122:499. doi: 10.1086/653086

Eales, S. A. (2015). Practical cosmology with lenses. Month. Notices RAS 446, 3224-3234. doi: 10.1093/mnras/stu2214

Enia, A., Negrello, M., Gurwell, M., Dye, S., Rodighiero, G., Massardi, M., et al. (2018). The Herschel-ATLAS: magnifications and physical sizes of 500- $\mu \mathrm{m}$-selected strongly lensed galaxies. Month. Notices RAS 475, 3467-3484. doi: 10.1093/mnras/sty021

Fan, X.-L., and Wu, Q. (2018). The radio/gamma connection of blazars from high to low radio frequencies. Astrophys. J. 869:133. doi: 10.3847/1538-4357/aaeece

Franceschini, A., Toffolatti, L., Mazzei, P., Danese, L., and de Zotti, G. (1991). Galaxy counts and contributions to the background radiation from $1 \mu \mathrm{m}$ to 1000 mim. Astron. Astrophys. Suppl. 89, 285-310.

Fuhrmann, L., Angelakis, E., Zensus, J. A., Nestoras, I., Marchili, N., Pavlidou, V., et al. (2016). The F-GAMMA programme: multi-frequency study of active galactic nuclei in the Fermi era. Programme description and the first 2.5 years of monitoring. Astron. Astrophys. 596:A45. doi: 10.1051/0004-6361/2015 28034

Fujimoto, S., Ouchi, M., and Kohno, K. e. a. (2018). ALMA $26 \mathrm{Arcmin}^{2}$ Survey of GOODS-S at One Millimeter (ASAGAO): average morphology of high-z dusty star-forming galaxies in an exponential disk $(n \simeq 1)$. Astrophys. J. 861:7. doi: 10.3847/1538-4357/aac6c4

Galluzzi, V., Massardi, M., Bonaldi, A., Casasola, V., Gregorini, L., Trombetti, T., et al. (2018). Characterization of polarimetric and total intensity behaviour of a complete sample of PACO radio sources in the radio bands. Month. Notices RAS 475, 1306-1322. doi: 10.1093/mnras/stx3216

Galluzzi, V., Puglisi, G., Burkutean, S., Liuzzo, E., Bonato, M., Massardi, M., et al. (2019). ALMA Band 3 polarimetric follow-up of a complete sample of faint PACO sources. Month. Notices RAS. doi: 10.1093/mnras/stz1930

Galvin, T. J., Seymour, N., Marvil, J., Filipović, M. D., Tothill, N. F. H., McDermid, R. M., et al. (2018). The spectral energy distribution of powerful starburst galaxies - I. Modelling the radio continuum. Month. Notices RAS 474, 779-799. doi: $10.1093 / \mathrm{mnras} / \mathrm{stx} 2613$

Ghisellini, G., Nardini, M., Tagliaferri, J. G., Schady, P., Rau, A., Foschini, L., et al. (2013). High-redshift Fermi blazars observed by GROND and Swift. Month. Notices RAS 428, 1449-1459. doi: 10.1093/mnras/sts140

Ghisellini, G., Tagliaferri, G., Sbarrato, T., and Gehrels, N. (2015). SDSS J013127.34 - 032100.1: a candidate blazar with an 11 billion solar mass black hole at $z=$ 5.18. Month. Notices RAS 450, L34-L38. doi: $10.1093 / \mathrm{mnrasl} / \mathrm{slv042}$

Glenn, J., Conley, A., Béthermin, M., Altieri, B., Amblard, A., Arumugam, V., et al. (2010). HerMES: deep galaxy number counts from a $P(D)$ fluctuation analysis of SPIRE Science Demonstration Phase observations. Month. Notices RAS 409, 109-121. doi: 10.1111/j.1365-2966.2010.17781.x

Gómez-Guijarro, C., Riechers, D. A., Pavesi, R., Magdis, G. E., Leung, T. K. D., Valentino, F., et al. (2019). Confirming herschel candidate protoclusters from ALMA/VLA CO observations. Astrophys. J. 872:117. doi: $10.3847 / 1538-4357 / a b 002$ a

Gonzalez, A. H., Gettings, D. P., Brodwin, M., Eisenhardt, P. R. M., Stanford, S. A., Wylezalek, D., et al. (2019). The massive and distant clusters of WISE survey. I Survey overview and a catalog of $>2000$ galaxy clusters at $z \simeq 1$. Astrophys. J. Suppl. 240:33. doi: 10.3847/1538-4365/aafad2

González-Nuevo, J., Argüeso, F., López-Caniego, M., Toffolatti, L., Sanz, J. L., Vielva, P., et al. (2006). The Mexican hat wavelet family: application to pointsource detection in cosmic microwave background maps. Month. Notices RAS 369, 1603-1610. doi: 10.1111/j.1365-2966.2006.10442.x

González-Nuevo, J., Lapi, A., Fleuren, S., Bressan, S., Danese, L., De Zotti, G., et al. (2012). Herschel-ATLAS: toward a Sample of 1000 Strongly Lensed Galaxies. Astrophys. J. 749:65. doi: 10.1088/0004-637X/749/1/65

González-Nuevo, J., Suárez Gómez, S. L., Bonavera, L., Sánchez-Lasheras, F., Argüeso, F., Toffolatti, L., et al. (2019). SHALOS: statistical herschelATLAS lensed objects selection. arXiv[Preprint].arXiv:1903.06424. doi: 10.1051/0004-6361/201935475

Granot, J., and Sari, R. (2002). The shape of spectral breaks in gamma-ray burst afterglows. Astrophys. J. 568, 820-829. doi: 10.1086/338966

Greaves, J. S., and Holland, W. S. (2002). "Submillimetre polarization of M82 and the Galactic Center: implications for CMB polarimetry," in Astrophysical Polarized Backgrounds, volume 609 of American Institute of 
Physics Conference Series, eds S. Cecchini, S. Cortiglioni, R. Sault, and C. Sbarra, 267-270.

Greenslade, J., Clements, D. L., Cheng, T., De Zotti, G., Scott, D., Valiante, E., et al. (2018). Candidate high-z protoclusters among the Planck compact sources, as revealed by Herschel-SPIRE. Month. Notices RAS 476, 3336-3359. doi: $10.1093 / \mathrm{mnras} / \mathrm{sty} 023$

Gupta, N., Reichardt, C. L., Ade, P. A. R., Anderson, A. J., Archipley, M., Austermann, J. E., et al. (2019). Fractional polarisation of extragalactic sources in the 500-square-degree SPTpol survey. arXiv[Preprint].arXiv:1907.02156.

Hanany, S., Alvarez, M., Artis, E., Ashton, P., Aumont, J., Aurlien, R., et al. (2019). PICO: probe of inflation and cosmic origins. arXiv[Preprint].arXiv:1902.10541.

Hilton, M., Hasselfield, M., Sifón, C., Battaglia, N., Aiola, S., Bharadwaj, V., et al. (2018). The atacama cosmology telescope: the two-season ACTPol sunyaev-zeldovich effect selected cluster catalog. Astrophys. J. Suppl. 235:20. doi: 10.3847/1538-4365/aaa6cb

Ho, A. Y. Q., Phinney, E. S., Ravi, V., Kulkarni, S. R., Petitpas, G., Emonts, B., et al. (2019). AT2018cow: a luminous millimeter transient. Astrophys. J. 871:73. doi: 10.3847/1538-4357/aaf473

Ivison, R. J., Swinbank, A. M., Smail, I., Harris, A. I., Bussmann, R. S., Cooray, A., et al. (2013). Herschel-ATLAS: a binary HyLIRG pinpointing a cluster of starbursting protoellipticals. Astrophys. J. 772:137. doi: $10.1088 / 0004-637 \mathrm{X} / 772 / 2 / 137$

Jackson, N. (2008). Gravitational lenses and lens candidates identified from the COSMOS field. Month. Notices RAS 389, 1311-1318. doi: $10.1111 /$ j.1365-2966.2008.13629.x

Jacobs, C., Collett, T., Glazebrook, K., McCarthy, C., Qin, A. K., Abbott, T. M. C., et al. (2019). Finding high-redshift strong lenses in DES using convolutional neural networks. Month. Notices RAS 484, 5330-5349. doi: $10.1093 / \mathrm{mnras} / \mathrm{stz} 272$

King, A., and Pounds, K. (2015). Powerful outflows and feedback from active galactic nuclei. Annu. Rev. Astron. Astrophys. 53, 115-154. doi: 10.1146/annurev-astro-082214-122316

Lacaille, K., Chapman, S., Smail, I., Steidel, C., Blain, A., Geach, J., et al. (2018). Two sub-millimetre bright protoclusters bounding the epoch of peak star formation activity. arXiv[Preprint].arXiv:1902.10541.

Lacy, M., Mason, B., Sarazin, C., Chatterjee, S., Nyland, K., Kimball, A., et al. (2019). Direct detection of quasar feedback via the Sunyaev-Zeldovich effect. Month. Notices RAS 483, L22-L27. doi: 10.1093/mnrasl/sly215

Lähteenmäki, A., Järvelä, E., Ramakrishnan, V., Tornikoski, M., Tammi, J., Vera, R. J. C., et al. (2018). Radio jets and gamma-ray emission in radio-silent narrow-line Seyfert 1 galaxies. Astron. Astrophys. 614:L1. doi: 10.1051/0004-6361/201833378

Leisawitz, D., Amatucci, E., Carter, R., DiPirro, M., Flores, A., Staguhn, J., et al. (2018). "The origins space telescope: mission concept overview," in Space Telescopes and Instrumentation 2018: Optical, Infrared, and Millimeter Wave, volume 10698 of Society of Photo-Optical Instrumentation Engineers (SPIE) Conference Series, 1069815. doi: 10.1117/12.2313823

Li, R., Frenk, C. S., Cole, S., Gao, L., Bose, S., and Hellwing, W. A. (2016). Constraints on the identity of the dark matter from strong gravitational lenses. Month. Notices RAS 460, 363-372. doi: 10.1093/mnras/stw939

López-Caniego, M., Herranz, D., González-Nuevo, J., Sanz, J. L., Barreiro, R. B., Vielva, P., et al. (2006). Comparison of filters for the detection of point sources in Planck simulations. Month. Notices RAS 370, 2047-2063. doi: 10.1111/j.1365-2966.2006.10639.x

Mancuso, C., Lapi, A., Cai, Z.-Y., Negrello, M., De Zotti, G., Bressan, A., et al. (2015). Predictions for Ultra-deep Radio Counts of Star-forming Galaxies. Astrophys. J. 810:72. doi: 10.1088/0004-637X/810/1/72

Marscher, A. P., and Gear, W. K. (1985). Models for high-frequency radio outbursts in extragalactic sources, with application to the early 1983 millimeterto-infrared flare of 3C 273. Astrophys. J. 298, 114-127. doi: 10.1086/163592

Marsden, D., Gralla, M., Marriage, T. A., Switzer, E. R., Partridge, B., Massardi, M., et al. (2014). The atacama cosmology telescope: dusty star-forming galaxies and active galactic nuclei in the Southern survey. Month. Notices RAS 439, 1556-1574. doi: 10.1093/mnras/stu001

Massardi, M., Bonaldi, A., Negrello, M., Ricciardi, S., Raccanelli, A., and de Zotti, G. (2010). A model for the cosmological evolution of low-frequency radio sources. Month. Notices RAS 404, 532-544. doi: 10.1111/j.1365-2966.2010.16305.x
Maturi, M., Bellagamba, F., Radovich, M., Roncarelli, M., Sereno, M., Moscardini, L., et al. (2019). AMICO galaxy clusters in KiDS-DR3: sample properties and selection function. Month. Notices RAS 485, 498-512. doi: 10.1093/mnras/stz294

Mehrtens, N., Romer, A. K., Hilton, M., Lloyd-Davies, E. J., Miller, C. J., Stanford, S. A., et al. (2012). The XMM Cluster Survey: optical analysis methodology and the first data release. Month. Notices RAS 423, 1024-1052. doi: 10.1111/j.1365-2966.2012.20931.x

Melin, J.-B., Bartlett, J. G., Cai, Z.-Y., De Zotti, G., Delabrouille, J., Roman, M., et al. (2018a). Dust in galaxy clusters: modeling at millimeter wavelengths and impact on Planck cluster cosmology. Astron. Astrophys. 617:A75. doi: 10.1051/0004-6361/201732292

Melin, J.-B., Bonaldi, A., Remazeilles, M., Hagstotz, S., Diego, J. M., HernándezMonteagudo, C., et al. (2018b). Exploring cosmic origins with CORE: cluster science. J. Cosmol. Astropart. Phys. 4:019. doi: 10.1088/1475-7516/2018/04/019

Merloni, A., Predehl, P., Becker, W., Böhringer, H., Boller, T., Brunner, H., et al. (2012). eROSITA science book: mapping the structure of the energetic universe. arXiv[Preprint].arXiv:1209.3114.

Metzger, B. D., Williams, P. K. G., and Berger, E. (2015). Extragalactic synchrotron transients in the era of wide-field radio surveys. I. Detection rates and light curve characteristics. Astrophys. J. 806:224. doi: 10.1088/0004-637X/806/2/224

Miller, T. B., Chapman, S. C., Aravena, M., Ashby, M. L. N., Hayward, C. C., Vieira, J. D., et al. (2018). A massive core for a cluster of galaxies at a redshift of 4.3 . Nature 556, 469-472. doi: 10.1038/s41586-018-0025-2

Mocanu, L. M., Crawford, T. M., Vieira, J. D., Aird, K. A., Aravena, M., Austermann, J. E., et al. (2013). Extragalactic millimeter-wave point-source catalog, number counts and statistics from $771 \mathrm{deg}^{2}$ of the SPT-SZ survey. Astrophys. J. 779:61. doi: 10.1088/0004-637X/779/1/61

Nartallo, R., Gear, W. K., Murray, A. G., Robson, E. I., and Hough, J. H. (1998). A millimetre/submillimetre polarization survey of compact flat-spectrum radio sources. Month. Notices RAS 297, 667-686. doi: 10.1046/j.1365-8711.1998.01405.x

Natarajan, P., and Sigurdsson, S. (1999). Sunyaev-zeldovich decrements with no clusters? Month. Notices RAS 302, 288-292. doi: 10.1046/j.1365-8711.1999.02116.x

Nayyeri, H., Keele, M., Cooray, A., Riechers, D. A., Ivison, R. J., Harris, A. I., et al. (2016). Candidate gravitationally lensed dusty star-forming galaxies in the herschel wide area surveys. Astrophys. J. 823:17. doi: $10.3847 / 0004-637 X / 823 / 1 / 17$

Negrello, M., Amber, S., and Amvrosiadis, A. e. a. (2017a). The Herschel-ATLAS: a sample of $500 \mu \mathrm{m}$-selected lensed galaxies over $600 \mathrm{deg}^{2}$. Month. Notices RAS 465, 3558-3580. doi: 10.1093/mnras/stw2911

Negrello, M., Gonzalez-Nuevo, J., and De Zotti, G. e. a. (2017b). On the statistics of proto-cluster candidates detected in the Planck all-sky survey. Month. Notices RAS 470, 2253-2261. doi: 10.1093/mnras/stx1367

Negrello, M., Hopwood, R., and De Zotti, G. E. A. (2010). The detection of a population of submillimeter-bright, strongly lensed galaxies. Science 330:800. doi: $10.1126 /$ science. 1193420

Negrello, M., Hopwood, R., Dye, S., da Cunha, E., Serjeant, S., Fritz, J., et al. (2014). Herschel *-ATLAS: deep HST/WFC3 imaging of strongly lensed submillimetre galaxies. Month. Notices RAS 440, 1999-2012. doi: 10.1093/mnras/ stu 413

Negrello, M., Perrotta, F., and González-Nuevo, J. E. A. (2007). Astrophysical and cosmological information from large-scale submillimetre surveys of extragalactic sources. Month. Notices RAS 377, 1557-1568. doi: $10.1111 / j .1365-2966.2007 .11708 . x$

Oteo, I., Ivison, R. J., Dunne, L., Manilla-Robles, A., Maddox, S., Lewis, A. J. R., et al. (2018). An extreme protocluster of luminous dusty starbursts in the early universe. Astrophys. J. 856:72. doi: 10.3847/1538-4357/aaalf1

Overzier, R. A. (2016). The realm of the galaxy protoclusters. A review. Astron. Astrophys. Rev. 24:14. doi: 10.1007/s00159-016-0100-3

Planck Collaboration III (2018). Planck 2018 results. III. High Frequency Instrument data processing and frequency maps. arXiv[Preprint].arXiv:1807.06207.

Planck Collaboration Int. XXVII (2015). Planck intermediate results. XXVII. Highredshift infrared galaxy overdensity candidates and lensed sources discovered by Planck and confirmed by Herschel-SPIRE. Astron. Astrophys. 582:A30. doi: $10.1051 / 0004-6361 / 201424790$ 
Planck Collaboration Int. XXXIX (2016). Planck intermediate results. XXXIX. The Planck list of high-redshift source candidates. Astron. Astrophys. 596:A100. doi: $10.1051 / 0004-6361 / 201527206$

Planck Collaboration VI (2018). Planck 2018 results. VI. Cosmological parameters. arXiv[Preprint].arXiv:1807.06209.

Planck Collaboration XIII (2011). Planck early results. XIII. Statistical properties of extragalactic radio sources in the Planck Early Release Compact Source Catalogue. Astron. Astrophys. 536:A13. doi: 10.1051/0004-6361/201116471

Planck Collaboration XLV (2016). Planck intermediate results. XLV. Radio spectra of northern extragalactic radio sources. Astron. Astrophys. 596:A106. doi: $10.1051 / 0004-6361 / 201527780$

Planck Collaboration XVIII (2011). Planck early results. XVIII. The power spectrum of cosmic infrared background anisotropies. Astron. Astrophys. 536:A18. doi: 10.1051/0004-6361/201116461

Planck Collaboration XXVI (2016). Planck 2015 results. XXVI. The Second Planck Catalogue of Compact Sources. Astron. Astrophys. 594:A26. doi: 10.1051/0004-6361/201526914

Planck Collaboration XXVII (2016). Planck 2015 results. XXVII. The second Planck catalogue of Sunyaev-Zeldovich sources. Astron. Astrophys. 594:A27. doi: $10.1051 / 0004-6361 / 201525823$

Planck Collaboration XXX (2014). Planck 2013 results. XXX. Cosmic infrared background measurements and implications for star formation. Astron. Astrophys. 571:A30. doi: 10.1051/0004-6361/201322093

Platania, P., Burigana, C., De Zotti, G., Lazzaro, E., and Bersanelli, M. (2002). Sunyaev-Zel'dovich effect from quasar-driven blast waves. Month. Notices RAS 337, 242-246. doi: 10.1046/j.1365-8711.2002.05907.x

Remazeilles, M., Banday, A. J., Baccigalupi, C., Basak, S., Bonaldi, A., De Zotti, G., et al. (2018). Exploring cosmic origins with CORE: B-mode component separation. J. Cosmol. Astropart. Phys. 4:023. doi: $10.1088 / 1475-7516 / 2018 / 04 / 023$

Rigby, E. E., Argyle, J., Best, P. N., Rosario, D., and Röttgering, H. J. A. (2015). Cosmic downsizing of powerful radio galaxies to low radio luminosities. Astron. Astrophys. 581:A96. doi: 10.1051/0004-6361/2015 26475

Rykoff, E. S., Rozo, E., Hollowood, D., Bermeo-Hernandez, A., Jeltema, T., Mayers, J., et al. (2016). The RedMaPPer galaxy cluster catalog from DES science verification data. Astrophys. J. Suppl. 224:1. doi: 10.3847/0067-0049/224/1/1

Saikia, D. J., and Salter, C. J. (1988). Polarization properties of extragalactic radio sources. Annu. Rev. Astron. Astrophys. 26, 93-144. doi: 10.1146/annurev.aa.26.090188.000521

Seiffert, M., Borys, C., Scott, D., and Halpern, M. (2007). An upper limit to polarized submillimetre emission in Arp 220. Month. Notices RAS 374, 409414. doi: 10.1111/j.1365-2966.2006.11186.x

Serjeant, S. (2017). Strong gravitational lenses and multi-wavelength galaxy surveys with akari, herschel, SPICA and EUCLID. Publ. Kor. Astron. Soc. 32, 251-255. doi: 10.5303/PKAS.2017.32.1.251

Silk, J., and Mamon, G. A. (2012). The current status of galaxy formation. Res. Astron. Astrophys. 12, 917-946. doi: 10.1088/1674-4527/12/8/004

Somerville, R. S., and Davé, R. (2015). Physical models of galaxy formation in a cosmological framework. Annu. Rev. Astron. Astrophys. 53, 51-113. doi: 10.1146/annurev-astro-082812-140951

Spilker, J., and Nyland, K. (2018). "Characterizing feedback through molecular outflows across cosmic time," in Science with a Next Generation Very Large Array, volume 517 of Astronomical Society of the Pacific Conference Series, ed E. Murphy, 657.

Spilker, J. S., Aravena, M., and Béthermin, M. e. a. (2018). Fast molecular outflow from a dusty star-forming galaxy in the early Universe. Science 361, 1016-1019. doi: $10.1126 /$ science.aap 8900

Strandet, M. L., Weiss, A., De Breuck, C., Marrone, D. P., Vieira, J. D., Aravena, M., et al. (2017). ISM properties of a massive dusty star-forming galaxy discovered at $z \sim 7$. Astrophys. J. Lett. 842:L15. doi: 10.3847/2041-8213/aa74b0

Su, T., Marriage, T. A., Asboth, V., Baker, A. J., Bond, J. R., Crichton, D., et al. (2017). On the redshift distribution and physical properties of ACT-selected DSFGs. Month. Notices RAS 464, 968-984. doi: 10.1093/mnras/stw2334

Sunyaev, R. A., and Zeldovich, Y. B. (1972). The observations of relic radiation as a test of the nature of X-Ray radiation from the clusters of galaxies. Comm. Astrophys. Space Phys. 4:173.
Swinbank, A. M., Smail, I., Longmore, S., Harris, A. I., Baker, A. J., De Breuck, C., et al. (2010). Intense star formation within resolved compact regions in a galaxy at $z=2.3$. Nature $464,733-736$. doi: $10.1038 /$ nature 08880

Tinti, S., and De Zotti, G. (2006). Constraints on evolutionary properties of $\mathrm{GHz}$ Peaked Spectrum galaxies. Astron. Astrophys. 445, 889-899. doi: 10.1051/0004-6361:20053752

Treu, T. (2010). Strong lensing by galaxies. Annu. Rev. Astron. Astrophys. 48, 87-125. doi: 10.1146/annurev-astro-081309-130924

Trombetti, T., Burigana, C., De Zotti, G., Galluzzi, V., and Massardi, M. (2018). Average fractional polarization of extragalactic sources at Planck frequencies. Astron. Astrophys. 618:A29. doi: 10.1051/0004-6361/201732342

Tucci, M., Toffolatti, L., de Zotti, G., and Martínez-González, E. (2011). High-frequency predictions for number counts and spectral properties of extragalactic radio sources. New evidence of a break at $\mathrm{mm}$ wavelengths in spectra of bright blazar sources. Astron. Astrophys. 533:A57. doi: 10.1051/0004-6361/201116972

Vieira, J. D., Crawford, T. M., Switzer, E. R., Ade, P. A. R., Aird, K. A., Ashby, M. L. N., et al. (2010). Extragalactic millimeter-wave sources in South pole telescope survey data: source counts, catalog, and statistics for an 87 squaredegree field. Astrophys. J. 719, 763-783. doi: 10.1088/0004-637X/719/1/763

Vieira, J. D., Marrone, D. P., Chapman, S. C., De Breuck, C., Hezaveh, Y. D., Wei $\beta$, A., et al. (2013). Dusty starburst galaxies in the early Universe as revealed by gravitational lensing. Nature 495, 344-347. doi: 10.1038/nature12001

Viero, M. P., Wang, L., Zemcov, M., Addison, G., Amblard, A., Arumugam, V., et al. (2013). HerMES: cosmic infrared background anisotropies and the clustering of dusty star-forming galaxies. Astrophys. J. 772:77. doi: 10.1088/0004-637X/772/1/77

Wagner, C. R., Courteau, S., and Brodwin, M. e. a. (2017). The evolution of star formation activity in cluster galaxies over $0.15<z<1.5$. Astrophys. J. 834:53. doi: $10.3847 / 1538-4357 / 834 / 1 / 53$

Wang, T., Elbaz, D., Daddi, E., Finoguenov, A., Liu, D., Schreiber, C., et al. (2016). Discovery of a galaxy cluster with a violently starbursting core at $z=2.506$. Astrophys. J. 828:56. doi: 10.3847/0004-637X/828/1/56

Weiß, A., De Breuck, C., Marrone, D. P., Vieira, J. D., Aguirre, J. E., Aird, K. A., et al. (2013). ALMA redshifts of millimeter-selected galaxies from the SPT survey: the redshift distribution of dusty star-forming galaxies. Astrophys. $J$. 767:88. doi: 10.1088/0004-637X/767/1/88

Wen, Z. L., and Han, J. L. (2018). A sample of 1959 massive galaxy clusters at high redshifts. Month. Notices RAS 481, 4158-4168. doi: 10.1093/mnras/ sty 2533

Whitehorn, N., Natoli, T., Ade, P. A. R., Austermann, J. E., Beall, J. A., Bender, A. N., et al. (2016). Millimeter transient point sources in the SPTpol 100 square degree survey. Astrophys. J. 830:143. doi: 10.3847/0004-637X/830/2/143

Willis, J. P., Clerc, N., Bremer, M. N., Pierre, M., Adami, C., Ilbert, O., et al. (2013). Distant galaxy clusters in the XMM Large Scale Structure survey. Month. Notices RAS 430, 134-156. doi: 10.1093/mnras/sts540

York, T., Jackson, N., Browne, I. W. A., Koopmans, L. V. E., McKean, J. P., Norbury, M. A., et al. (2005). CLASS B0631+519: last of the Cosmic Lens All-Sky Survey lenses. Month. Notices RAS 361, 259-271. doi: $10.1111 /$ j.1365-2966.2005.09170.x

Zavala, J. A., Montaña, A., Hughes, D. H., Yun, M. S., Ivison, R. J., Valiante, E., et al (2018). A dusty star-forming galaxy at $z=6$ revealed by strong gravitational lensing. Nat. Astron. 2, 56-62. doi: 10.1038/s41550-017-0297-8

Conflict of Interest Statement: The authors declare that the research was conducted in the absence of any commercial or financial relationships that could be construed as a potential conflict of interest.

Copyright (c) 2019 De Zotti, Bonato, Negrello, Trombetti, Burigana, Herranz, López-Caniego, Cai, Bonavera and González-Nuevo. This is an open-access article distributed under the terms of the Creative Commons Attribution License (CC BY). The use, distribution or reproduction in other forums is permitted, provided the original author(s) and the copyright owner(s) are credited and that the original publication in this journal is cited, in accordance with accepted academic practice. No use, distribution or reproduction is permitted which does not comply with these terms. 